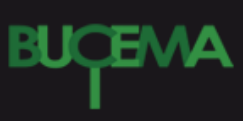

Bulletin du centre d'études médiévales d'Auxerre | BUCEMA

Hors-série $n^{\circ} 6 \mid 2013$

Autour du cloître : les chapelles Notre-Dame et les accès au chapitre

\title{
L'église Sainte-Marie et le chapitre à Castelletto Cervo et dans les monastères clunisiens de l'Italie du Nord
}

Eleonora Destefanis

\section{OpenEdition}

\section{Journals}

Édition électronique

URL : https://journals.openedition.org/cem/12698

DOI : $10.4000 /$ cem. 12698

ISSN : 1954-3093

Éditeur

Centre d'études médiévales Saint-Germain d'Auxerre

Référence électronique

Eleonora Destefanis, «L'église Sainte-Marie et le chapitre à Castelletto Cervo et dans les monastères clunisiens de l'Italie du Nord », Bulletin du centre d'études médiévales d'Auxerre / BUCEMA [En ligne], Horssérie $n^{\circ} 6$ | 2013, mis en ligne le 19 avril 2013, consulté le 03 mars 2023. URL : http:// journals.openedition.org/cem/12698; DOI : https://doi.org/10.4000/cem.12698

Ce document a été généré automatiquement le 3 mars 2023

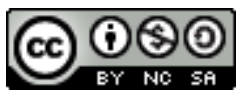

Creative Commons - Attribution - Pas d'Utilisation Commerciale - Partage dans les Mêmes Conditions 4.0 International - CC BY-NC-SA 4.0

https://creativecommons.org/licenses/by-nc-sa/4.0/ 


\section{L'église Sainte-Marie et le chapitre à Castelletto Cervo et dans les monastères clunisiens de l'Italie du Nord}

\section{Eleonora Destefanis}

1 La bibliographie récente a bien mis en évidence que l'affirmation du monachisme clunisien dans l'Italie du Nord, avec une concentration en Lombardie actuelle et plus généralement dans le nord-ouest de la péninsule, n'est pas un phénomène homogène, tant $d u$ point de vue chronologique que géographique ${ }^{1}$.

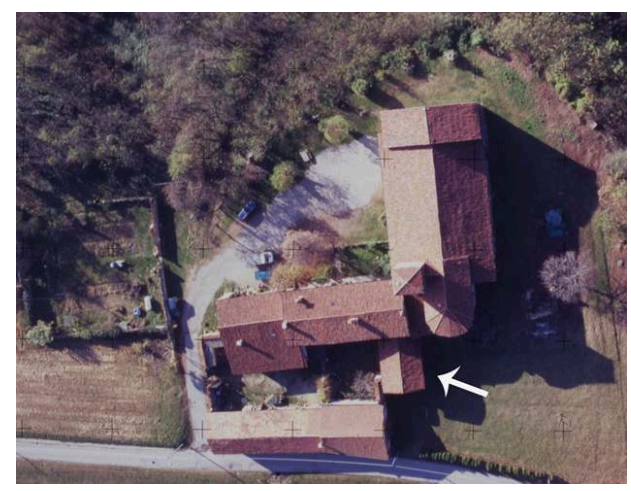


Fig. 1 - Carte de la présence clunisienne dans I'Italie du Nord (Centre et Ouest, $\mathrm{x}^{\mathrm{e}}$-XII ${ }^{\mathrm{e}}$ siècles).

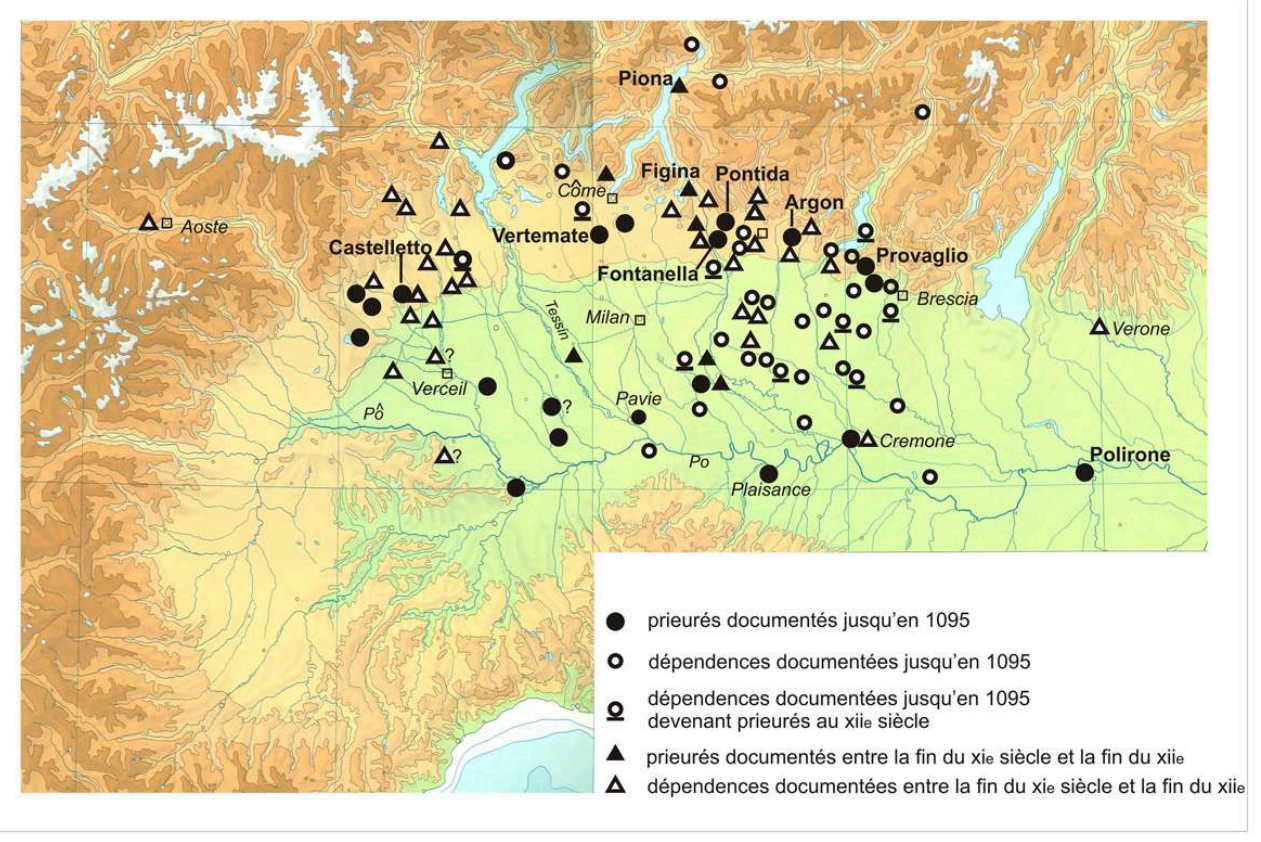

2 Le véritable essor de l'implantation clunisienne débute un siècle après la fondation du premier prieuré clunisien par Mayeul à Pavie à la fin des années 960, et plus encore à partir de 1076, époque de la "querelle des investitures». Les tensions entre le pouvoir impérial et pontifical, à l'intérieur même de l'épiscopat de l'Italie du Nord affaiblissent l'institution épiscopale, créant ainsi des conditions favorables à l'affirmation de l'abbaye bourguignonne. C'est justement à cette époque, entre la seconde moitié du $\mathrm{XI}^{\mathrm{e}}$ siècle et le $\mathrm{XII}^{\mathrm{e}}$ siècle, que l'on trouve les premiers bâtiments connus de sites appartenant au monde clunisien. En ce qui concerne les structures matérielles, les travaux de Paolo Piva et la récente synthèse d'Anna Segagni constituent des points de repère essentiels ${ }^{2}$ pour dresser un tableau, auquel les fouilles du prieuré de Castelletto Cervo au Piémont, qui viennent de se conclure, apportent une importante contribution, illustrée ci-dessous.

3 L'un des aspects sur lesquels s'est concentrée jusqu'à maintenant l'attention des spécialistes est le rapport entre les solutions architecturales adoptées dans les prieurés de la région (et dans l'abbaye de Polirone) et Cluny - essentiellement le Cluny de Conant $^{3}$ - et, plus largement, en Bourgogne ${ }^{4}$. À ce propos, après plus d'un siècle de discussions entre les spécialistes ${ }^{5}$, les approches historiographiques sont, aujourd'hui encore, assez différentes et la question reste ouverte. Par exemple, Anna Segnani, dans son tableau dressé en 2010, insiste sur les rapports des ensembles architecturaux clunisiens italiens avec le contexte local, sans nier les contacts avec le monde transalpin, non seulement pour ce qui concerne les techniques de constructions, mais également pour les choix de projet et l'organisation des formes et volumes ${ }^{6}$.

Les églises des principaux prieurés italiens présentent des éléments communs et assez répandus - montrant néanmoins à leur tour plusieurs variantes -, comme l'a mis en évidence Paolo Piva. Pour sa part, il propose d'aller au-delà du faux problème d'une " architecture clunisienne" pour l'Italie du nord, et s'interroge sur des "schémas fonctionnels » - à la fois pour les abbayes, les prieurés et leurs dépendances - liés à des exigences liturgiques différenciées ${ }^{7}$. Parmi ces éléments, on distingue, entre autres, la 
nef à trois vaisseaux de trois ou quatre travées, le transept non débordant, haut ou bas (parfois il s'agit d'un "pseudo-transept ", complètement invisible de l'extérieur), la croisée, carrée ou rectangulaire, surmontée par une tour-clocher octogonale parfois sur coupole, et surtout le soi-disant "plan bénédictin", comportant un chevet à trois absides et des collatéraux ouverts sur le sanctuaire. L'apparition de ces éléments architecturaux, bien attestés en Bourgogne et en milieu clunisien, est assez précoce dans les prieurés italiens. Pour cette raison et afin d'expliquer ce rayonnement, on a fréquemment évoqué le rôle de "médiation " que doivent avoir joué certains prieurs dont la biographie montre des rapports très étroits avec Cluny, tels Alberto da Prezzate (fondateur de Pontida et impliqué dans la fondation de plusieurs autres prieurés en Lombardie) ${ }^{8}$ ou Gérard, qui aurait participé à la fondation de Vertemate et Cantù, en provenance de la Charité-sur-Loire et de Joigny ${ }^{9}$. Bien conscients de la nécessité de disposer d'ensembles architecturaux répondant à des exigences liturgiques précises, ils auraient ainsi imposé la création de ceux que Piva appelle des «spazi funzionali» auxquels on ne pouvait pas renoncer ${ }^{10}$. Dans cette perspective et en refusant tout automatisme dans la transmission des modèles, il souligne plutôt la reprise transversale des formules de la tradition monastique plus ancienne, réinterprétées en milieu clunisien pour fournir des schémas fonctionnels à l'implantation des nouveaux prieurés et ainsi capables de s'adapter aux situations spécifiques ${ }^{11}$.

5 Même pour l'Italie, l'un des aspects pour lesquels cette «marque » clunisienne serait plus nettement visible est sans doute représenté par l'église secondaire, très souvent mais pas exclusivement - dédiée à la Vierge, placée au sud-est de l'édifice de culte principal et en rapport direct avec la salle capitulaire ${ }^{12}$. Cette chapelle semble constituer un véritable « édifice identitaire ", selon la définition de Nicolas Reveyron ${ }^{13}$, et sa disposition topographique en rapport avec le cloître (répondant à des raisons à la fois pratiques et liturgiques) devient parfois l'élément sur lequel se fonde l'identification d'un établissement monastique avec un prieuré clunisien.

6 C'est, entre autres, le cas de Saint-Nicolas de Piona, sur le lac de Côme, qui est en effet l'un des rares contextes où subsistent des structures appartenant à une chapelle flanquant l'église principale.

7 On ne connaît pas la date de la fondation du prieuré qui est attesté seulement en 1195 et en 1204. Cependant, un monasterium de Piona, est documenté sur le site en 1169 (mais l'expression Sancti Nicolai de Piona est déjà mentionnée dans une liste des propriétés foncières en 1154) ${ }^{14}$. L'ensemble monastique présente deux églises (fig. 2)

8 - la principale, Saint-Nicolas ${ }^{15}$, de forme trapézoïdale, très allongée $(27,60 \mathrm{~m}$ de longueur), révèle au moins deux phases de construction: nef courte vers l'est et première abside ; allongement de la nef et réfection de l'abside ;

9 - au sud-est, sur un éperon surélevé, un second bâtiment cultuel est attesté par une abside de 3,58 m de large (dimensions retrouvées à l'intérieur de l'abside même) (fig. 3). 
Fig. 2 - Piona, prieuré Saint-Nicolas. Sur la gauche : plan de l'ensemble (d'après C. MARCoRA, II Priorato di Piona, Lecco, 1972, p. 61) ; sur la droite: plan du prieuré en 1722, Archives d'État de Como, Fondo U.T.E., Catasto teresiano, Mappe, Olgiasca con Piona, cart. 117, mappa di prima stazione (Piona), f' 3-4, (autorisation prot. n. 1669/31.13.04.01, 2 août 2012, parere n. 8/2012. "Riproduzione vietata").
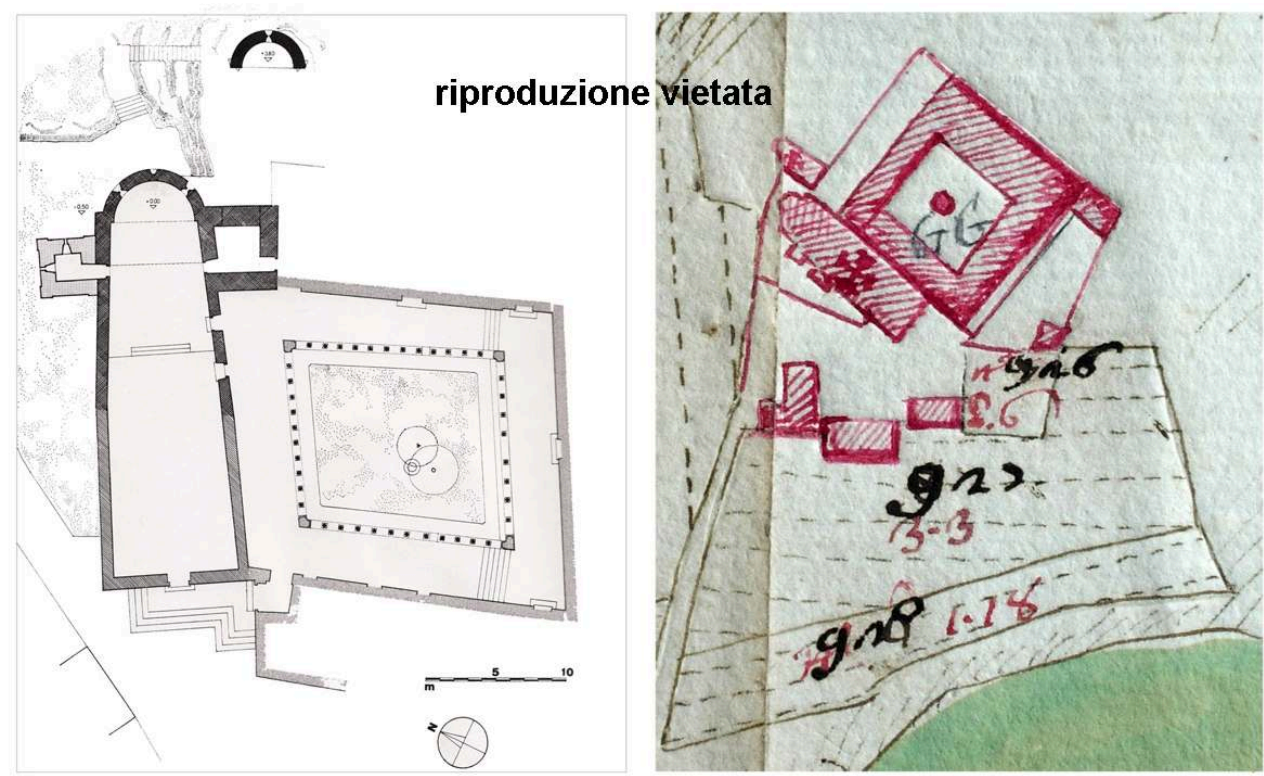

Le cadastre dit teresiano, datant de 1722 (fig. 2), montre néanmoins d'une façon plus complète ce bâtiment, à une seule nef, de $13 \mathrm{~m}$ sur $4,30 \mathrm{~m}$ environ ${ }^{16}$.

Fig. 3 - Piona. Le chevet de l'église Saint-Nicolas et l'abside de Sainte-Marie (d'après P. PIVA, Architettura monastica nell'Italia del Nord. Le chiese cluniacensi, Milano, 1998, p. 96).

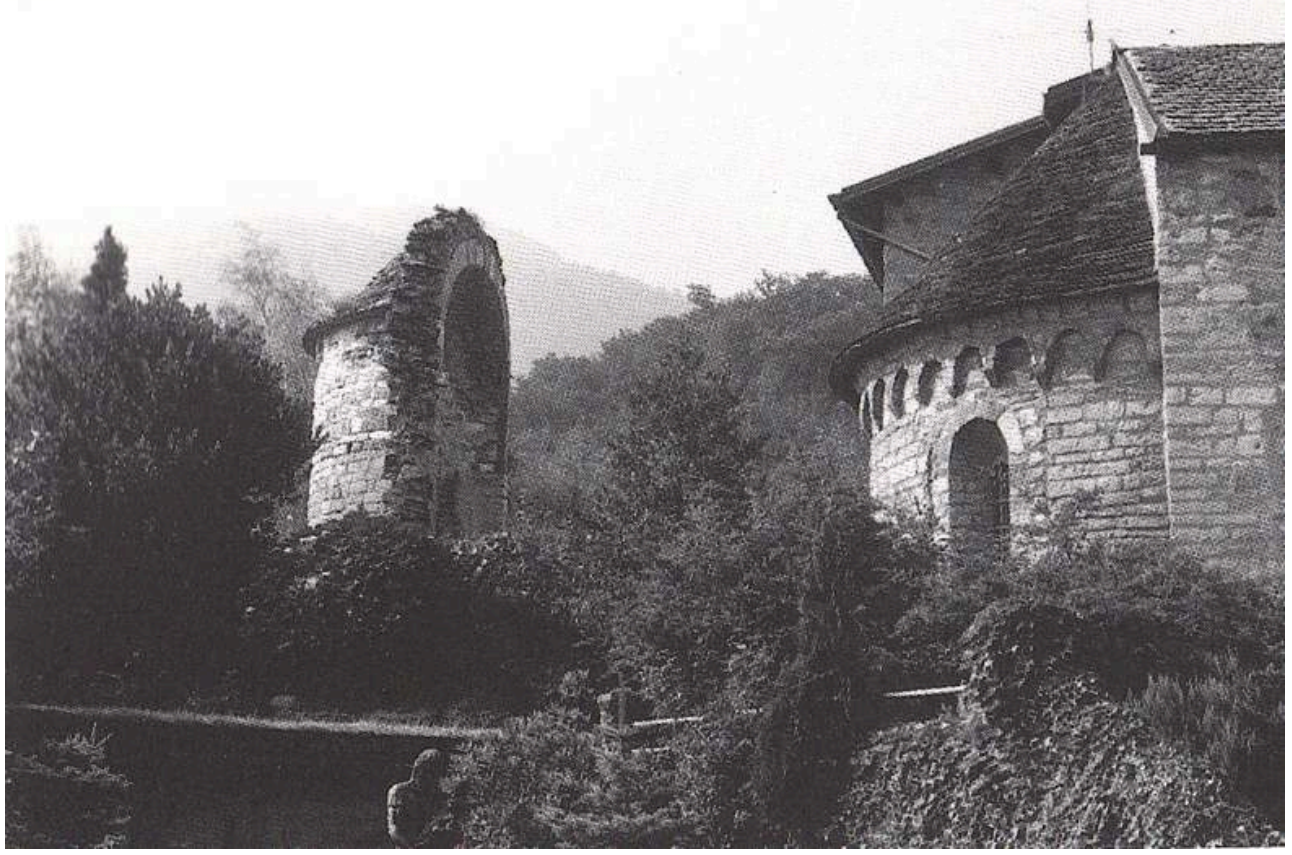

11 Cette abside, que la technique de construction permet de rattacher à la deuxième phase de l'église principale, est datée de la fin $\mathrm{du}_{\mathrm{XI}}^{\mathrm{e}}$-premières décennies du XII ${ }^{\mathrm{e}}$ siècle, tandis 
que la première implantation de l'ensemble des bâtiments est attribuée au troisième quart du XI ${ }^{\mathrm{e}}$ siècle ou, plus probablement, peu après. La présence de cette chapelle, dont les sources d'époque moderne relatent la dédicace mariale ${ }^{17}$, est reconnue comme étant de plan typiquement clunisien. Par rapport à la grande église, et bien qu'elles soient situées sur deux niveaux différents, la chapelle a été directement rapprochée, pour sa forme et sa position, à l'église Sainte-Marie I de Cluny, datée, selon Conant, de la première moitié du $\mathrm{XI}^{\mathrm{e}}$ siècle. Selon Piva, et au-delà de la question de la réelle correspondance avec une réalité clunisienne, l'existence de la chapelle représenterait en soi un élément qui, face aux informations éparses et un peu ambiguës des sources écrites, permettrait de reconnaître sur le site, au moins à la fin $\mathrm{du}_{\mathrm{Xl}}^{\mathrm{e}}$ siècle, non seulement la présence d'un monastère, mais, bien plus précisément, l'implantation d'un prieuré clunisien, peut-être fondé sur l'initiative de Pontida. L'allongement vers l'ouest de l'église Saint-Nicolas de 16 à presque 28 mètres irait d'ailleurs dans ce sens, en suggérant un besoin accru de place aussi bien qu'un rôle différent de l'institution.

En l'état actuel des connaissances, on ne peut rien dire sur le rapport entre l'église Sainte-Marie et le cloître, daté à ce jour du XIII ${ }^{e}$ siècle. La différence de niveau, 3,80 m, mentionnée sur un plan de $1972{ }^{18}$, pose un problème, encore accentué par la présence, sur le côté sud du sanctuaire, de la base quadrangulaire du clocher précédant l'actuel. Il a aujourd'hui disparu, mais une source du XVII siècle le décrit comme octogonal dans son élévation ${ }^{19}$, ce qui ne semble pas exclure une datation à l'époque romane. L'absence de données d'archéologie du bâti sur cette pièce et les importants travaux de restauration pendant le $\mathrm{xx}^{\mathrm{e}}$ siècle ne permettent pas pour l'instant de résoudre la question de l'aménagement de ce point de connexion entre la chapelle et l'aile est du cloître ${ }^{20}$. Néanmoins, le cadastre de 1722 établit d'une façon très claire le rapport direct entre ces deux bâtiments ${ }^{21}$, bien que la différence de niveau entre eux reste un problème. On se demande à ce propos si la communication pouvait se faire à un niveau haut - à l'étage, en supposant l'existence d'une porte s'ouvrant dans la façade de la chapelle -, en utilisant, pour atteindre la salle capitulaire, un escalier dans la pièce qualifiée de clocher à l'époque moderne; ou faut-il plus simplement imaginer un système d'escalier (couvert?) à l'extérieur, dans l'angle entre l'église secondaire et l'aile du cloître? 
Fig. 4 - Argon, prieuré Saint-Paul. Plan de l'ensemble en 1500 environ, Archives d'État de Brescia, Ospedale Maggiore di Brescia, fondo S. Eufemia, b. 18, dis. n. 100 (d'après P. PIVA, Architettura monastica nell'Italia del Nord. Le chiese cluniacensi, Milano, 1998, p. 138).

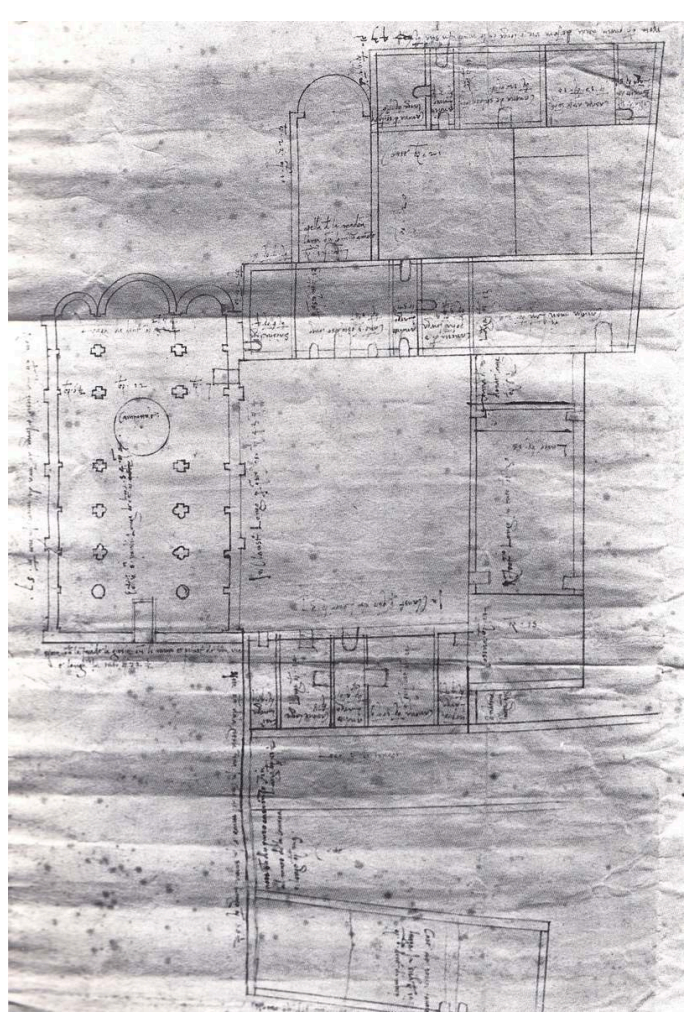

Quoique dépourvu presque intégralement de structures matérielles, le prieuré d'Argon, dans la région de Bergame, est en revanche bien attesté par un important document iconographique datant de 1500 environ (fig. 4). Conservé aux Archives d'État de Brescia, il restitue l'organisation du monastère avant la reconstruction de la fin $\mathrm{du} \mathrm{XVII}^{\mathrm{e}}$-début $\mathrm{XVIII}^{\mathrm{e}}$ siècle et garde la mémoire de la situation à l'époque romane ${ }^{22}$. La fondation est attestée en 1079, quand le comte de Bergame, Giselbert, donne des terres à Cluny pour l'édification d'un monastère dédié à Paul et à Pierre ${ }^{23}$. Sur le côté sud de l'église principale, le plan montre un cloître présentant, dans son aile orientale, une séquence de pièces que Piva identifie respectivement comme la sacristie - communiquant avec le collatéral sud du sanctuaire -, la salle capitulaire, aisément reconnaissable par le portail central flanqué par deux ouvertures (probablement des fenêtres), un passage étroit et deux autres pièces dans lesquelles il propose de reconnaître l'auditorium et la camera. Immédiatement à l'est de la salle capitulaire et communiquant avec celle-ci par une porte légèrement désaxée, s'élève une chapelle (dont la légende indique la dédicace à Sainte-Marie), à nef unique avec une abside dont le plan mentionne les dimensions extérieures de 12,54 m x 6,60 m.

Une petite cour, au sud de la chapelle, était accessible par le couloir au milieu de l'aile est du cloître, juste à côté de la salle capitulaire, comme on vient de le voir. Sur l'autre côté de la cour, accolé à l'extrémité est de l'aula de la chapelle, un bâtiment est représenté. $\mathrm{Au} \mathrm{XVI}^{\mathrm{e}}$ siècle, il était destiné au personnel laïque et organisé à son tour avec une séquence de pièces (le plan indique, pour la pièce contiguë à l'église, une caneva - dépôt/construction utilitaire - de sec[u]larii) ; un passage étroit, comme celui que l'on voit à côté de la salle capitulaire, permettait la communication entre la petite cour et l'extérieur. Du point de vue fonctionnel, la destination de cet édifice à l'époque 
romane reste inconnue. Piva hésite à l'identifier à l'infirmerie (bien que la position par rapport à ce que l'on sait de Cluny semblerait compatible), étant donné qu'à la fin du $\mathrm{xv}^{\mathrm{e}}$ siècle, les moines d'Argon n'étaient que sept, ce qui exclurait l'exigence d'un bâtiment construit pour cet usage ${ }^{24}$. Néanmoins, il faut d'abord considérer qu'il s'agit d'une mention très tardive, qui ne reflet pas forcement le nombre effectif des moines aux origines de la communauté; en tout cas, on ne peut pas mettre forcement en relation la taille du monastère et la construction de bâtiments "spécialisés » : en effet, même chez un petit noyau de religieux (et Argon n'est pas, d'ailleurs, un prieuré secondaire dans la Lombardie médiévale), peuvent être bien présentes des exigences à la fois liturgiques, pratiques, mais peut-être aussi idéologiques, liées à la volonté de reproduire de plus ou moins près la situation de Cluny.

Le problème de la datation de l'ensemble se pose, faute de témoignages matériels, très réduits pour le site. Piva date la partie orientale de l'église selon la première mention documentaire (vers 1080), tout en soulignant la correspondance du plan de la chapelle avec la première chapelle Sainte-Marie de Cluny (avant 1085). Toujours selon les reconstructions de Conant, il en place l'édification au tout début de l'histoire du monastère, en proposant de l'identifier comme la seule église existante au moment de la fondation, utilisée par les moines pendant la construction de la prieurale : en tout cas, son usage définitif pour la communauté en tant que second sanctuaire aurait été prévu dès l'origine.

Une reconstruction semblable a été supposée par le même auteur pour les chapelles d'autres prieurés italiens, tels que Castelletto, sur lequel nous reviendrons, et Vertemate, situé entre Milan et Côme. Sa fondation date de $1084{ }^{25}$ et apparaît strictement liée à celle d'Argon. Le prieur d'Argon, David, aurait en effet appelé Gérard, d'origine milanaise, mais probablement moine à Cluny et, comme on l'a déjà dit, prieur de la Charité-sur-Loire et de Joigny, pour guider la construction du nouveau monastère dans la région de Côme. Les nombreux donateurs offrent à Cluny un «castrum unum juris nostris ", à l'intérieur duquel se trouve un monastère dédié à la Sainte-Croix, une dédicace qui, quelques années après (1093), est remplacée par celle de Saint JeanBaptiste. Le site a connu, dès les années 1960, d'importantes restaurations/ reconstructions, qui ont concerné l'église et surtout le cloître, détruit par la commune de Côme en 1287, cloître rebâti sur les fondations retrouvées ${ }^{26}$. L'aile est de celui-ci montre encore quelques traces des maçonneries et des ouvertures romanes, qui semblent suggérer une organisation assez proche de celle qu'on vient de voir pour Argon. Du nord au sud (fig. 5) une sacristie, dont on conserve le portail permettant l'accès au collatéral sud du sanctuaire, la salle capitulaire - dont subsiste le portail ouest, avec des fenêtres symétriques sur les deux côtés, et le portail est - et peut-être, le couloir et d'autres pièces (auditorium et camera?). L'église communiquait avec le cloître par deux portails, l'un (originel) dans le collatéral sud, l'autre (de restauration) dans le transept. 
Fig. 5 - Vertemate, prieuré Saint-Jean Baptiste. Sur la gauche : plan de l'ensemble et hypothèse d'emplacement de la possible église secondaire (d'après P. PIVA, Architettura monastica nell'Italia del Nord. Le chiese cluniacensi, Milano, 1998, p. 140) ; sur la droite : le côté est de l'aile orientale du cloître, avec le portail de la salle capitulaire (cl. E. Destefanis).
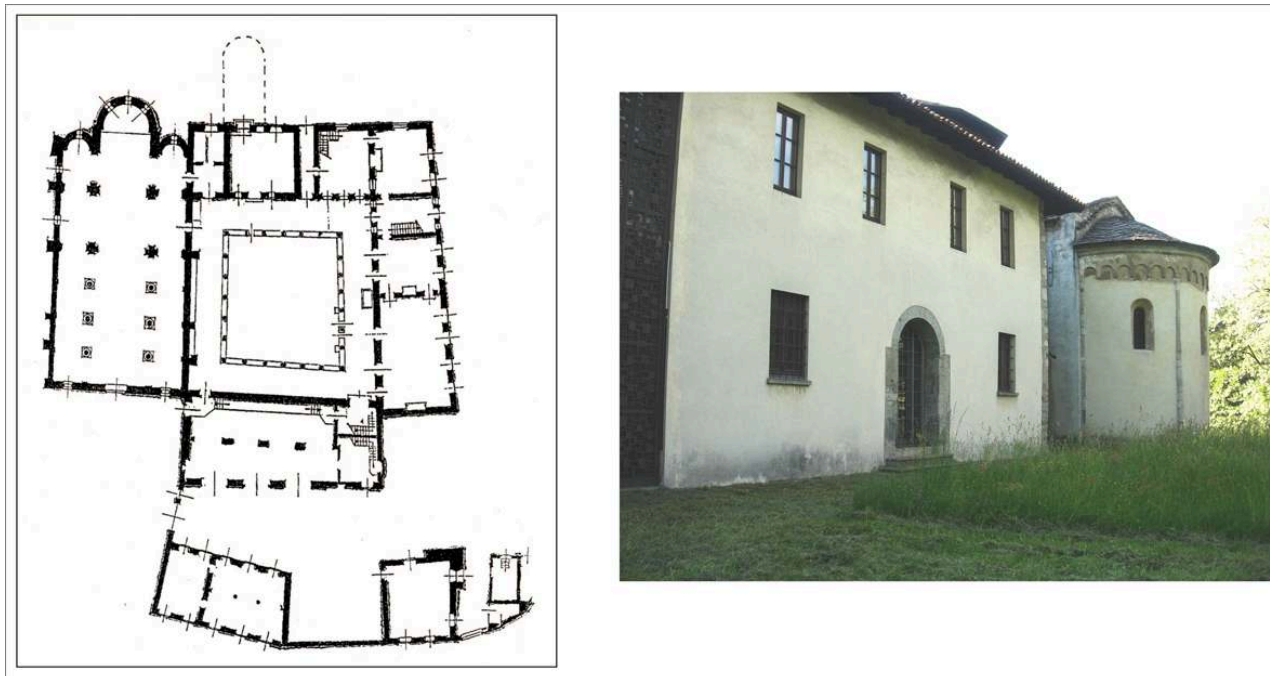

17 En se fondant sur les ressemblances qui semblent lier Vertemate à Argon et surtout à cause de la présence du portail est de la salle capitulaire, Piva pense, pour ce site, à l'existence d'une deuxième église derrière et contiguë à la salle du chapitre. Il s'agit, peut-être, là aussi, de l'oratoire Sainte-Croix mentionné en 1084, qui serait le premier lieu de culte du monastère, en usage pendant la construction de la grande prieurale, dédiée à Saint-Jean et qui aurait, enfin, imposé sa dédicace. 
Fig. 6 - Figina, prieuré Saint-Nicolas. Plan de l'église en 1571 environ, Archives de l'Archevêché de Milan, sez. X, Visite pastorali, Pieve di Oggiono, vol. 1, q. 5 (d'après P. PIVA, Architettura monastica nell'Italia del Nord. Le chiese cluniacensi, Milano, 1998, p. 139).

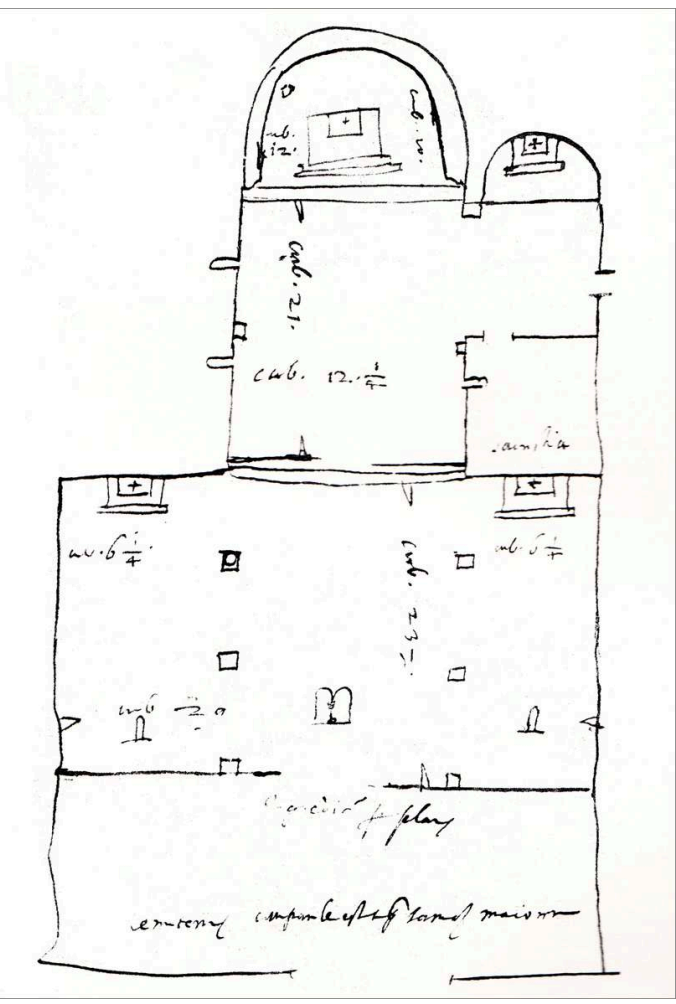

Parfois les sources écrites donnent des informations qui ne sont pas faciles à traduire du point de vue topographique et architectural. Dans le cas de Saint-Nicolas de Figina, fondé près du lac de Côme en 1107 par la veuve milanaise Contessa ${ }^{27}$, une reconstruction de l'époque moderne a presque entièrement effacé l'état d'origine de la seconde décennie du XII ${ }^{\mathrm{e}}$ siècle. Il subsiste encore des traces de l'église (piliers de la nef centrale), un bâtiment à trois nefs se terminant avec trois absides, tel que le suggère encore un plan de 1571 environ, conservé aux Archives de l'Archevêché de Milan (fig. 6). Le Liber Notitiae Sanctorum Mediolani, datant de la période entre 1304 et 1311, relate la présence de deux autels très vraisemblablement à placer dans les absides latérales, dédiées à Saint-Martin et à la Vierge ${ }^{28}$. Un autel, probablement situé dans le collatéral nord, ferait de cette pièce, selon Piva, une sorte de chapelle mariale incorporée dans l'église, en sauvegardant ainsi un élément « marqueur » de l'adhésion au monde clunisien. Le Liber Notitiae mentionne aussi une ecclesia sancti Sigismundi ${ }^{29}$, que la tradition locale situe en relation avec le cimetière, placé, au moins à l'époque moderne, devant l'église «lungo un lato della medesima " (sur un côté de celle-ci) ${ }^{30}$. Cette indication a suggéré à Piva la possibilité d'une position au sud-est du bâtiment cultuel principal ${ }^{31}$. Dans ce cas, en suivant cette reconstruction, on envisagerait un double renvoi à Cluny: d'un côté il y aurait une correspondance "topographique", avec l'église au sud-est (consacrée à un saint), de l'autre une liaison " de dédicace », qui serait assurée par un collatéral-chapelle mariale à l'intérieur de l'église. C'est d'ailleurs la situation qu'il semble relever aussi à Vertemate, où à la plausible chapelle au sud-est de la prieurale s'ajoute la présence - probable, bien qu'attestée seulement par des sources de l'époque moderne - d'une chapelle avec un autel dédié à la Vierge dans le collatéral nord ${ }^{32}$. 
Selon Piva, une question, liée à l'idée de la création d'une "chapelle mariale clunisienne » à l'intérieur de l'église, se pose pour certains aspects semblables surtout pour les prieurés «mineurs " qui n'auraient pas développé un oratoire autonome ${ }^{33}$, comme le monastère de Saint-Pierre de Provaglio, sur le lac d'Isée, fondé en 1083 par Ambrosius et Opprandus de loco Tocingo ${ }^{34}$, membres d'une famille de l'aristocratie locale. Ici, une récente étude archéologique des maçonneries et une fouille, impliquant l'église et une partie du cloître $d u \mathrm{XVI}^{\mathrm{e}}$ siècle, ont permis de mettre en évidence plusieurs phases de la construction du prieuré (fig. 7).

Fig. 7 - Provaglio d'Iseo, prieuré Saint-Pierre-in-Lamosa. Plan avec phasage et reconstructions de l'église : fin XIe et fin XII ${ }^{e}$-début XIII ${ }^{e}$ siècle (d'après BREDA A., VALSECCHI A., " San Pietro in Lamosa. La vicenda architettonica tra Medioevo e Rinascimento ", in F. SINA, A. VALSECCHI (dir.), San Pietro in Lamosa in Provaglio d'Iseo. Storia e arte, Provaglio d'Iseo, 2007, p. 25 et 36).
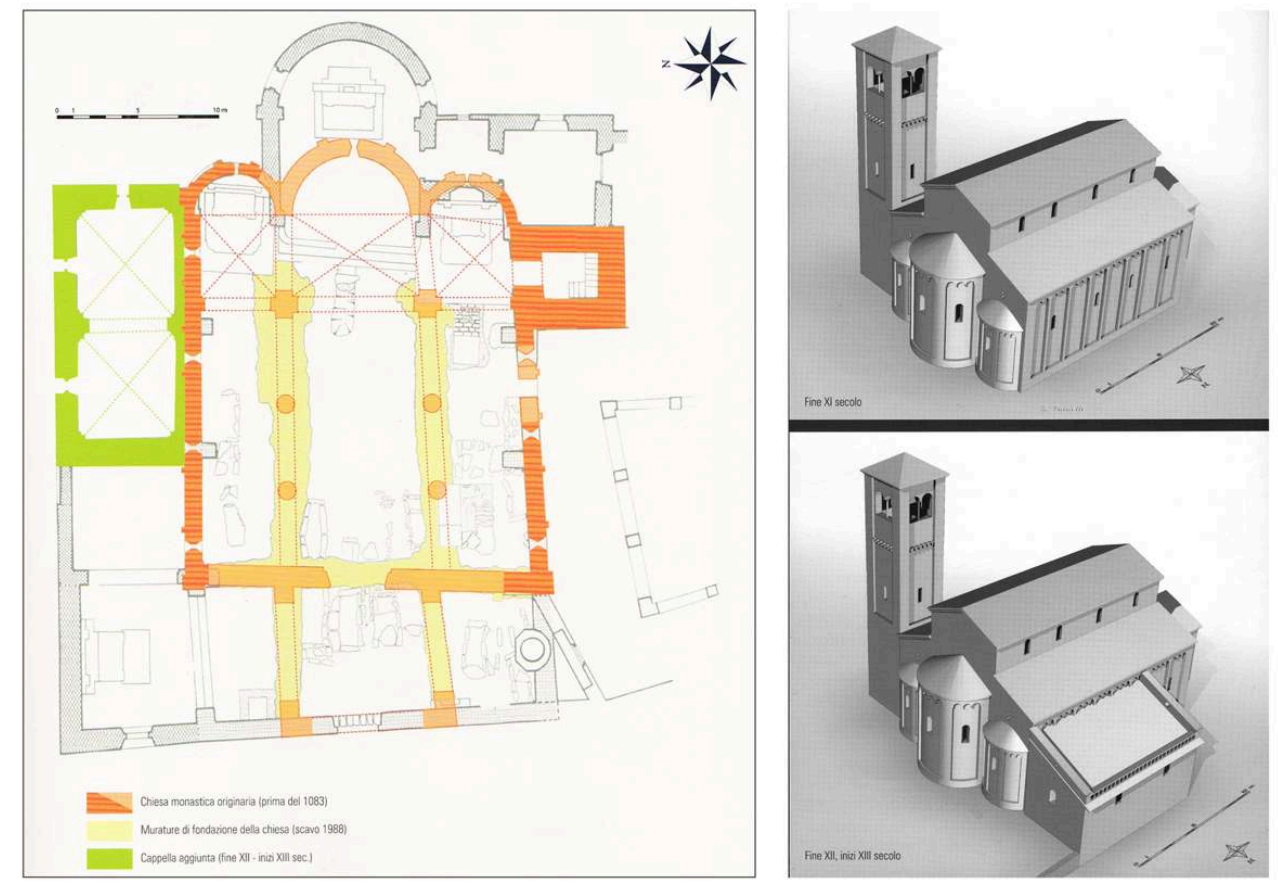

Le bâtiment cultuel se présente, à la fin du xi siècle, comme une implantation à trois vaisseaux précédés par un petit porche en correspondance de la nef centrale et flanquées au sud par un clocher. À la fin $d u x{ }^{e}$-début xiII ${ }^{e}$ siècle une chapelle rectangulaire est ajoutée, s'appuyant contre le mur gouttereau nord. Une porte devait permettre la communication avec le chœur, bien qu'aujourd'hui la situation soit assez différente, à cause de l'ouverture, à l'époque moderne, de deux grandes arcades qui ont fait disparaitre une partie importante de l'élévation du mur nord de l'église ${ }^{35}$. Piva a identifié cet oratoire comme la chapelle mariale du prieuré. Cette fonction aurait été, à l'origine, associée au collatéral nord, où la présence d'un autel de la Vierge pourrait être suggérée par l'image d'une figure féminine auréolée (fig. 8), peinte au-dessus d'une arcade, tout près de l'absidiole nord et à côté d'une baie de l'église romane, le long de l'ancien mur gouttereau nord de celle-ci, percé par une grande arcade du Xve siècle ${ }^{36}$. L'image a été reconnue par Piva comme une Annonciation et datée aux XIII ${ }^{\mathrm{e}}$-XIV ${ }^{\mathrm{e}}$ siècles ; plus récemment, en revanche, on a laissé ouverte la possibilité de l'identifier génériquement à une sainte, en proposant une datation aux XII ${ }^{\mathrm{e}}-\mathrm{XIII}^{\mathrm{e}}$ siècles ${ }^{37}$. 
21 La question soulève, généralement, des points d'interrogation, tant du point de vue architectural que liturgique. À Provaglio, au-delà du problème posé par le déplacement d'un pôle cultuel aussi important du collatéral, à côté du maître-autel, vers une chapelle latérale, il faudrait se demander jusqu'à quel point l'emplacement d'un autel de la Vierge dans une abside latérale peut être qualifié de "typiquement clunisien " (en tant que « substitut » de la chapelle mariale au sud-est de l'église principale), étant bien attesté dans la tradition monastique depuis le haut Moyen Âge. En même temps, comme Éric Palazzo l'a bien montré, le culte de Marie s'impose progressivement à cette époque : les dispositifs liturgiques - et les autels en particulier - « semblent calqués sur le modèle de la Hiérarchie céleste ", où le rôle de la Vierge devient de plus en plus important et l'autel dont elle est la dédicataire « occupe aussi cette place centrale dans l'organisation d'un espace liturgique et sa définition ecclésiologique ». Cet autel devient ainsi fréquemment le principal autel secondaire, placé à côté de celui du Sauveur, dans le transept ou dans l'axe longitudinal de la nef, jusqu'à arriver, à l'époque romane, à la création d'une véritable "architecture mariale ", où la Vierge est parfois vénérée dans un espace qui lui est entièrement dédié, une crypte ou une rotonde ${ }^{38}$.

Les lieux où se déroule cette vénération dans les espaces ecclésiaux sont différents et correspondent à des significations différentes, qui ne sont pas si facilement « interchangeables ». En restant en milieu clunisien, le cas même de Castelletto (sur lequel on reviendra) semble d'ailleurs le suggérer, où Marie est à la fois la dédicataire d'une chapelle autonome au sud-est de l'église principale et d'un autel dans la prieurale (situé dans un collatéral, comme à Fontanella ?), bien qu'attesté seulement à l'époque moderne ${ }^{39}$. 
Fig. 8 - Provaglio d'Iseo. La fresque représentant une figure féminine avec auréole (Sainte Marie?), sur le mur gouttereau nord de l'église romane (d'après BREDA A., VALSECCHI A., " San Pietro in Lamosa. La vicenda architettonica tra Medioevo e Rinascimento ", in F. SINA, A. VALSECCHI (dir.), San Pietro in Lamosa in Provaglio d'Iseo. Storia e arte, Provaglio d'Iseo, 2007, p. 31).

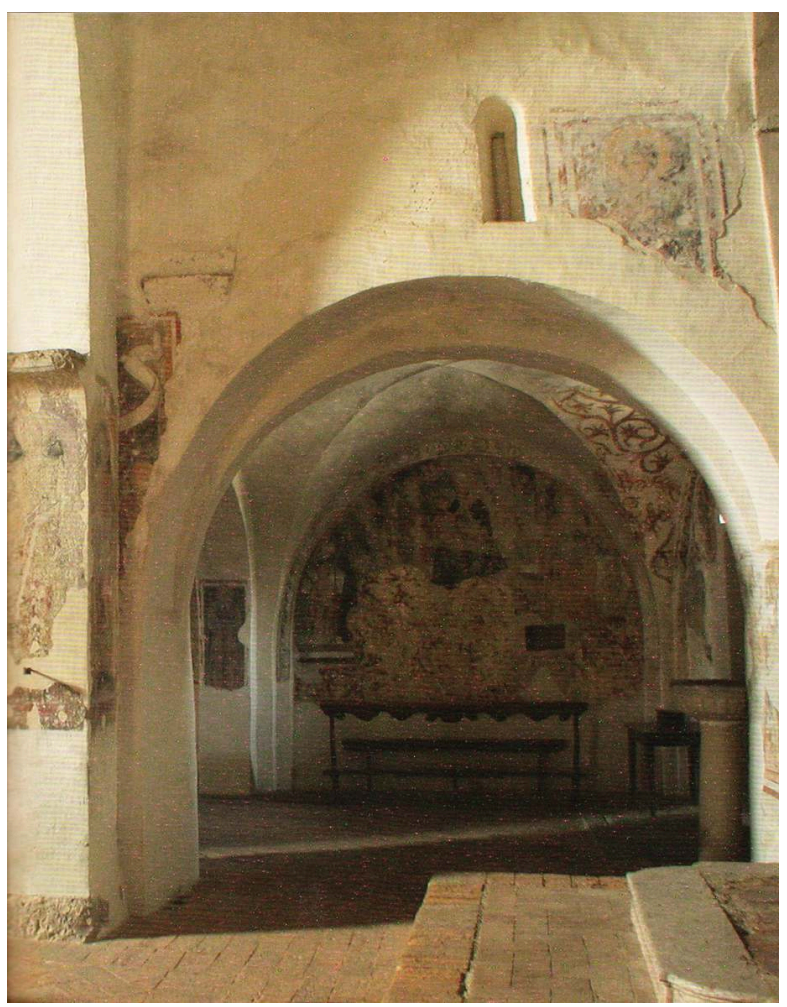

Le cas du prieuré de Saint-Egidius de Fontanella, près de Pontida, vient illustrer à son tour ces observations. Fondé en 1080 par Alberto da Prezzate ${ }^{40}$, le site conserve l'église qui montre plusieurs états de construction, situés entre 1080 et le milieu du XII ${ }^{\mathrm{e}}$ siècle ${ }^{41}$. $\mathrm{Au}$-delà des différentes propositions de phasage, les interprétations récentes partagent l'idée d'un premier bâtiment à nef unique, avec un transept en saillie débouchant sur trois absides (plan en « $\mathrm{T} »)$, intégré successivement dans une église à trois vaisseaux avec un transept non débordant et un sanctuaire à collatéraux (correspondant au premier transept); une tour quadrangulaire sur coupole recouvre la croisée du transept de la dernière phase romane. Comme dans d'autres cas, même à Fontanella, une source de 1308 atteste la présence d'un autel dédié à la Vierge ${ }^{42}$, à situer dans un des collatéraux du sanctuaire.

Un portail ouvert dans le collatéral sud et appartenant à la phase la plus ancienne, datée de la fin $\mathrm{du}_{\mathrm{XI}}^{\mathrm{e}}$ siècle, reliait le bâtiment cultuel à ceux qui abritaient les moines, dont quelques traces subsistent dans les édifices qui occupèrent successivement l'espace du cloître. En particulier, une restitution récemment proposée a mis en évidence un bâtiment rectangulaire parallèle à l'église d'origine et situé à $18 \mathrm{~m}$ environ au sud de celle-ci, conservant des baies, associées, pour des raisons stylistiques, à la première phase du monastère. 
Fig. 9 - Fontanella, prieuré Saint-Egidius. Plan de l'ensemble et hypothèse d'emplacement de la possible église secondaire (d'après P. PIVA, Architettura monastica nell'Italia del Nord. Le chiese cluniacensi, Milano, 1998, p. 60).

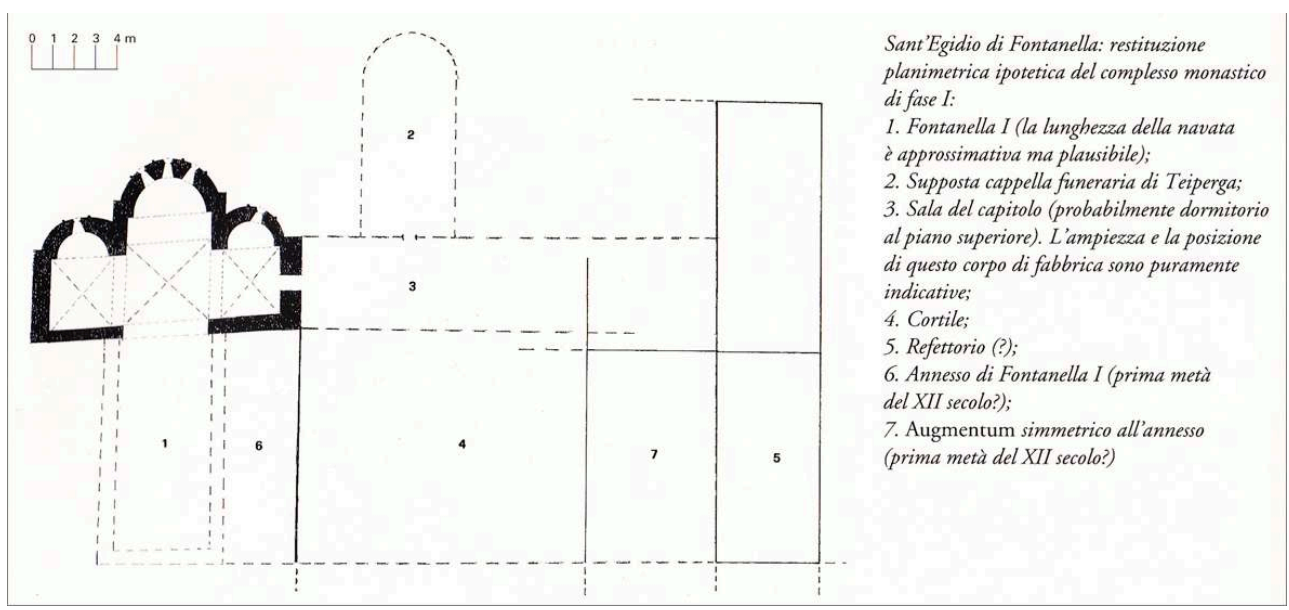

À ce cloître, qui semble s'organiser progressivement pendant les transformations romanes successives, Paolo Piva relève la présence d'une chapelle, qu'il situe à côté du chapitre, au sud-est de l'église (fig. 9). Cet oratoire aurait abrité, au moins à l'époque moderne, le sépulcre de Sainte Toperge, évoquée dans l'acte de 1080 où Alberto da Prezzate souligne que la donation à Cluny pour la constitution du monastère est faite pro anima de lui-même et de trois autres personnages, parmi lesquels figure la sainte ${ }^{43}$. Le corps de la sainte, enseveli dans le prieuré, devait devenir très tôt un objet de vénération, puisque, déjà, l'inventaire de 1308 rappelle que "in ecclesia et apud sepulturam beate Toperge » brûlaient huit lampes ${ }^{44}$.

L'hypothèse de Piva, qui laisse entrevoir une autre église possible placée dans la disposition "clunisienne » - de dédicace inconnue, faisant quand même pendant avec la « chapelle mariale » de la prieurale -, se fonde en particulier sur le Rotulus bonorum de 1536, qui mentionne effectivement le lien étroit entre la capella, abritant le sépulcre de la sainte, et la salle capitulaire ${ }^{45}$. Les indications de la source ne sont pourtant pas si nettes à propos de sa localisation et l'interprétation suscite des doutes, surtout si l'on considère un inventaire des biens de 1498 qui parle aussi de la capella avec le tombeau de Toperge, mais qui la place "dentro lo claustro » et non à l'extérieur (côté est) de celui-ci ${ }^{46}$, laissant ainsi supposer une collocation différente et soulevant d'importants problèmes lexicaux au moment où l'on cherche à traduire en structures matérielles le terme capella employé par la source.

La question reste ouverte. Ces mentions sont en tout cas assez tardives; il faut surtout garder à l'esprit que la source la plus ancienne mentionnant le sépulcre de la sainte l'inventaire de 1308 cité ci-dessus -, bien que très précise dans la liste des biens et des objets appartenant aux différents bâtiments du monastère, systématiquement nommés (sagrestia, dormitorio, camera domini prioris, coquina monasterii...), ne mentionne pas un édifice de culte secondaire, abritant le sépulcre de la donatrice/fondatrice, évoqué, en revanche, tout seul. Certes, les argumenta ex silentio ont une valeur très relative, sans considérer que, de toute façon, c'est le corps saint qui constitue le focus de la vénération et de l'intérêt de l'écrivain. Il reste quand même le soupçon d'une position différente du tombeau, peut-être, comme le suppose Marta Spini, à l'intérieur de l'église prieurale ${ }^{47}$ ou dans les galeries du cloître ${ }^{48}$, où l'on pourrait penser à une monumentalisation successive. 
Le problème d'une chapelle située au sud-est de l'église, liée au chapitre et ayant une fonction funéraire et de lieu de commémoration pour le fondateur/bienfaiteur suggérée par Piva pour Fontanella ${ }^{49}$, évoque de près également l'abbaye Saint-Benoît de Polirone, située dans une ancienne insula du Pô, au sud-est de Mantoue. Fondation de la famille des marquis de Canossa du début du XI ${ }^{e}$ siècle (1007), le monastère est rattaché à Cluny en 1077, peu après la célèbre rencontre à laquelle participent la comtesse Mathilde, Grégoire VII, Henri IV et Hugues de Semur. Mathilde donne l'abbaye au pape, qui la soumet à Cluny. Polirone représente en réalité un cas à part dans le monde clunisien, à cause des conditions exceptionnelles dans lesquelles se réalise l'annexion à Cluny : elle est d'ailleurs la seule abbaye dans le panorama clunisien italien ${ }^{50}$.

Le site connut une imposante reconstruction au $\mathrm{xvI}^{\mathrm{e}}$ siècle et plusieurs travaux de restauration au cours du $\mathrm{Xx}^{\mathrm{e}}$ siècle, à l'occasion desquels on effectua des sondages non stratigraphiques qui posent de nombreux problèmes. Plus récemment, des fouilles, menées à plusieurs reprises et avec différentes interprétations des données, pendant les années 1990, attendent encore une publication définitive, tout comme les dernières explorations menées entre 2006 et 2008.

Fig. 10 - Polirone, abbaye Saint-Benoît. Sur la gauche : plan de l'ensemble avec une tentative de reconstruction des parcours liturgiques des moines; sur la droite : reconstruction de l'intérieur de l'église Sainte-Marie (d'après P. PIVA, « L'abbazia di Polirone nel XII secolo: architettura e vita monastica. Una lettura comparata della documentazione archeologica e scritta ", in A. C. QUINTAVALLE (dir.), Arredi liturgici e architettura, Milano, 2007, p. 57 et 69).
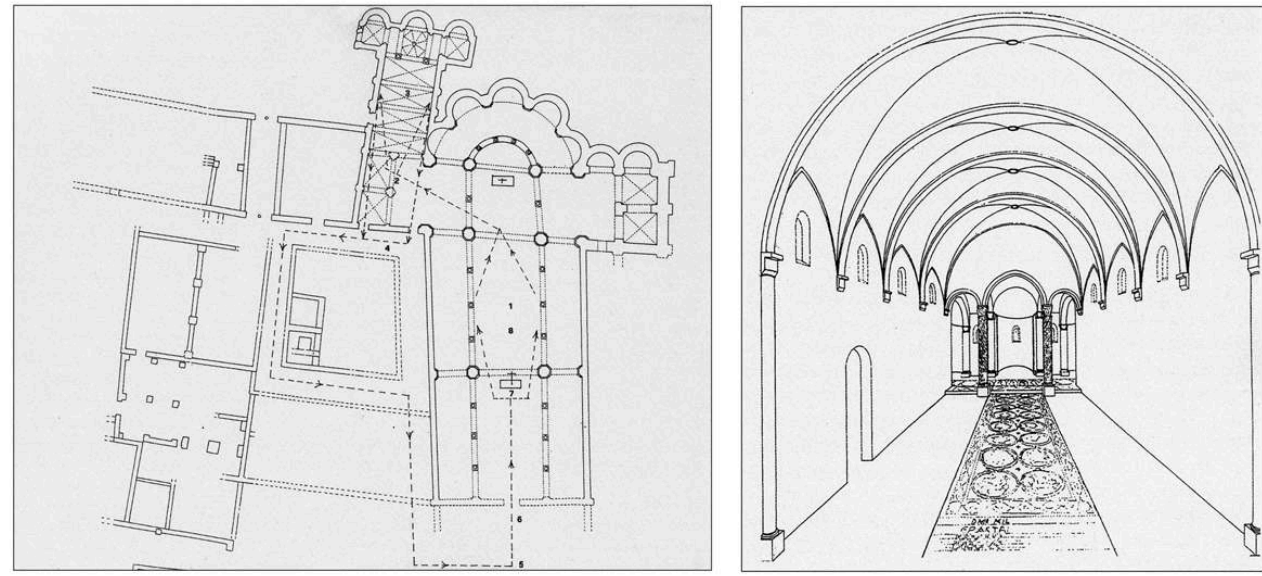

La situation est très complexe du point de vue de l'architecture; on se borne ici à exposer les derniers résultats des études très détaillées de Paolo Piva ${ }^{51}$, qui a proposé la reconstruction suivante (fig. 10). L'église, organisée en trois nefs, présentait vers l'est un déambulatoire à chapelles rayonnantes qui s'ouvrait sur un transept dont le bras sud était organisé en deux vaisseaux s'achevant chacun par une absidiole ; sur la travée dans l'angle sud-est se trouvait le clocher. Au bras nord - peut-être organisé sur deux vaisseaux et quatre travées lui aussi, - était liée la chapelle Sainte-Marie, qui présente une seule nef, voûtée, probablement dès l'origine, avec des arêtes sur des travées rectangulaires très allongées. Elle se termine par un transept débordant, qui prévoit deux « passages » voûtés en berceau, situés entre les bras - voûtés à leur tour d'arêtes et la croisée, couverte par une coupole sur laquelle s'élevait une tour octogonale. Trois absides achevaient l'église vers l'est, tandis que la nef débouchait sur le transept par un arc triple sur deux colonnes. Un portail s'ouvrait sur le côté nord, à la hauteur de la 
deuxième travée, tandis qu'à l'ouest la chapelle s'ouvrait sur le transept au moyen de deux grands arcs, aujourd'hui bouchés, mais encore décelables. Le sol du transept conserve une mosaïque, datée par une inscription de 1151, tandis que dans la nef, des morceaux d'une mosaïque vraisemblablement contemporaine indiquent la présence d'une sorte de couloir central décoré, sur les deux côtés duquel étaient probablement disposés les sièges des moines.

La chronologie des deux églises reste très problématique. Carlo Arturo Quintavalle, en observant la divergence d'orientation entre la partie est et les vaisseaux de l'abbatiale, songeait à plusieurs phases de construction, en proposant pour ceux-ci une datation de la moitié du XI ${ }^{\mathrm{e}}$ siècle. Ils auraient été suivis, à la fin du XI ${ }^{\mathrm{e}}$ siècle, par la réalisation de la chapelle mariale, tandis que la dernière phase (déambulatoire et transept) se situerait déjà au XII ${ }^{\mathrm{e}}$ siècle ${ }^{52}$. Piva, en reprenant toute la question, pense en revanche à un seul chantier ${ }^{53}$; il n'a en effet décelé aucun témoignage évident d'une succession de phases dans les maçonneries (il s'est toutefois appuyé principalement sur les observations de l'architecte qui avait conduit les travaux de restauration, dans un contexte de données qui ne sont plus contrôlables). La datation proposée est la période 1115 (année de la mort de Mathilde)-1140, en pensant donc à une reconstruction globale portant notamment sur les églises : en suivant le modèle de la chapelle Sainte-Marie de Cluny ("phase II»), cette importante intervention aurait été focalisée sur un oratoire funéraire «clunisien» dans lequel aurait pu être ensevelie la grande protectrice de l'abbaye.

Fig. 11 - Polirone. Aile est du cloître : la salle capitulaire (sur la droite) et les pièces contigües au XII siècle, selon les fouilles récentes (d'après S. LUSUARDI SIENA, C. GIOSTRA, « San Benedetto Po: l'abbazia di Matilde di Canossa. Archeologia di un grande monastero dell'Europa benedettina ", in M. C. SOMmA (dir.), Cantieri e maestranze nell'Italia medievale, Actes du Colloque (Chieti-San Salvo, 16-18 mai 2008), Spoleto, 2010, p. 491).

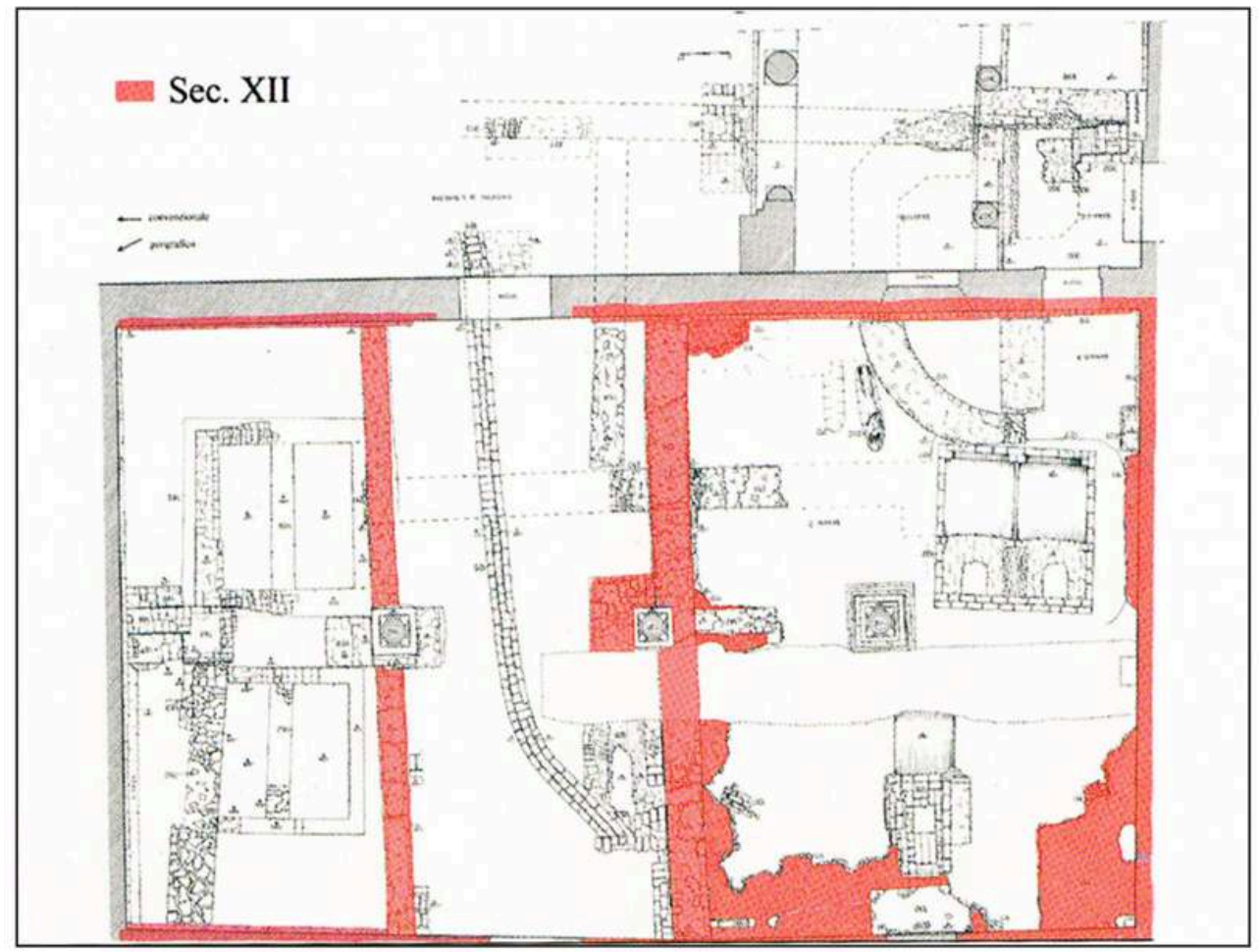



dont on garde encore quelques traces, s'appuie la salle capitulaire du XII ${ }^{e}$ siècle (environ $12 \mathrm{~m} \times 9 \mathrm{~m}$ ) (fig. 11), qui a été fouillée et qui s'ouvrait sur la galerie est du cloître par un portail (constitué en partie par des éléments de remploi) et deux fenêtres juxtaposées, dont subsiste une colonne. La fouille menée dans cette aile a aussi permis de mettre en évidence, immédiatement au nord de la salle du chapitre, un passage qui met en communication le cloître avec un espace à l'est (et donc au nord de la chapelle SainteMarie), mentionné dans la documentation plus tardive comme le claustrum infirmorum ${ }^{54}$. C'est dans cet ensemble, partiellement reconstruit par l'archéologie surtout pour ce qui concerne le cloître - que se déroule la liturgie processionnelle décrite par les Consuetudines du XII ${ }^{\mathrm{e}}$ siècle, qui prévoyait plusieurs stationes, et dans laquelle la chapelle Sainte-Marie jouait un rôle très complexe (fig. 10).

Au-delà des grandes transformations que la plupart des monastères clunisiens norditaliens ont connu, la difficulté d'interprétation des données est liée à une pratique de l'archéologie du bâti et de fouille stratigraphique qui n'est pas encore répandue concernant ces sites. C'est en revanche dans cette perspective que l'on a abordé la recherche à Castelletto Cervo, situé à $30 \mathrm{~km}$ environ au nord-ouest de Verceil. Du prieuré médiéval (fig. 12), mentionné pour la première fois en $1092{ }^{55}$, subsistent plusieurs édifices en élévation et d'autres ont été retrouvés à l'occasion d'une fouille qui a démarré en 2009 (et qui vient de se conclure, ce qui rend provisoire le tableau que l'on présente ci-dessous) ${ }^{56}$.

Fig. 12 - Castelletto Cervo, prieuré Saint-Pierre-et-Paul. L'ensemble monastique (cl. G. Viazzo) ; en évidence : l'église Sainte-Marie.

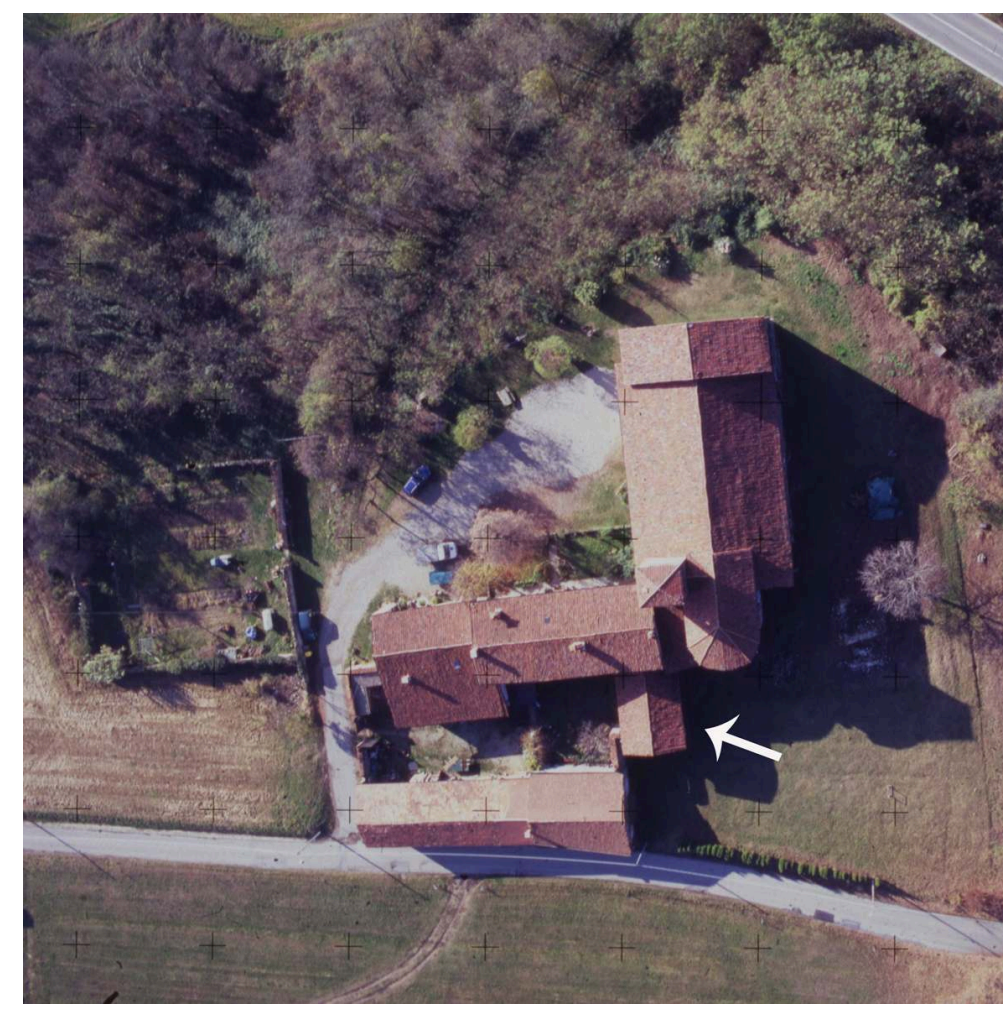

L'église, à l'exception du chœur, des voûtes et de la création des pièces sur les bascôtés, garde l'implantation romane - en trois nefs, transept non débordant, sanctuaire

Bulletin du centre d'études médiévales d'Auxerre | BUCEMA, Hors-série nº 6 | 2013 
et collatéraux ouverts sur celui-ci - remontant peut-être à la fin du $\mathrm{XI}^{\mathrm{e}}$ siècle, notamment en ce qui concerne la partie orientale. Les absides ont disparu, mais la fouille a permis de retrouver une partie de celle du nord, suffisamment étendue pour en proposer une restitution. Il s'agit probablement d'un chantier long, qui se termine vers l'ouest avec une façade bien conservée, qui montre déjà des caractères typiques du $\mathrm{XII}^{\mathrm{e}}$ siècle. Une surélévation du vaisseau central semble avoir été réalisée vers le milieu $\mathrm{du} \mathrm{XII}^{\mathrm{e}}$ siècle, dans un contexte de monumentalisation qui comporte aussi une réfection de la couverture, avec la construction de grands arcs doubleaux, comme celui que l'on voit dans les combles, engagé dans la contre-façade. Vraisemblablement pendant la première moitié du même siècle se met en place la construction d'un cloître à galeries, dont l'aile est subsiste en partie en élévation, tandis que l'aile ouest et un petit tronçon de l'aile sud ont été retrouvés en fouille, au niveau des fondations ${ }^{57}$ (fig. 13).

Fig. 13 - Castelletto Cervo. Ortophotographie des fouilles 2009-2011 (à l'exception de l'aire au nord de l'avant-nef) (plan G. Viazzo, photomosaïque N. Pozzato, réelaboration E. Destefanis).

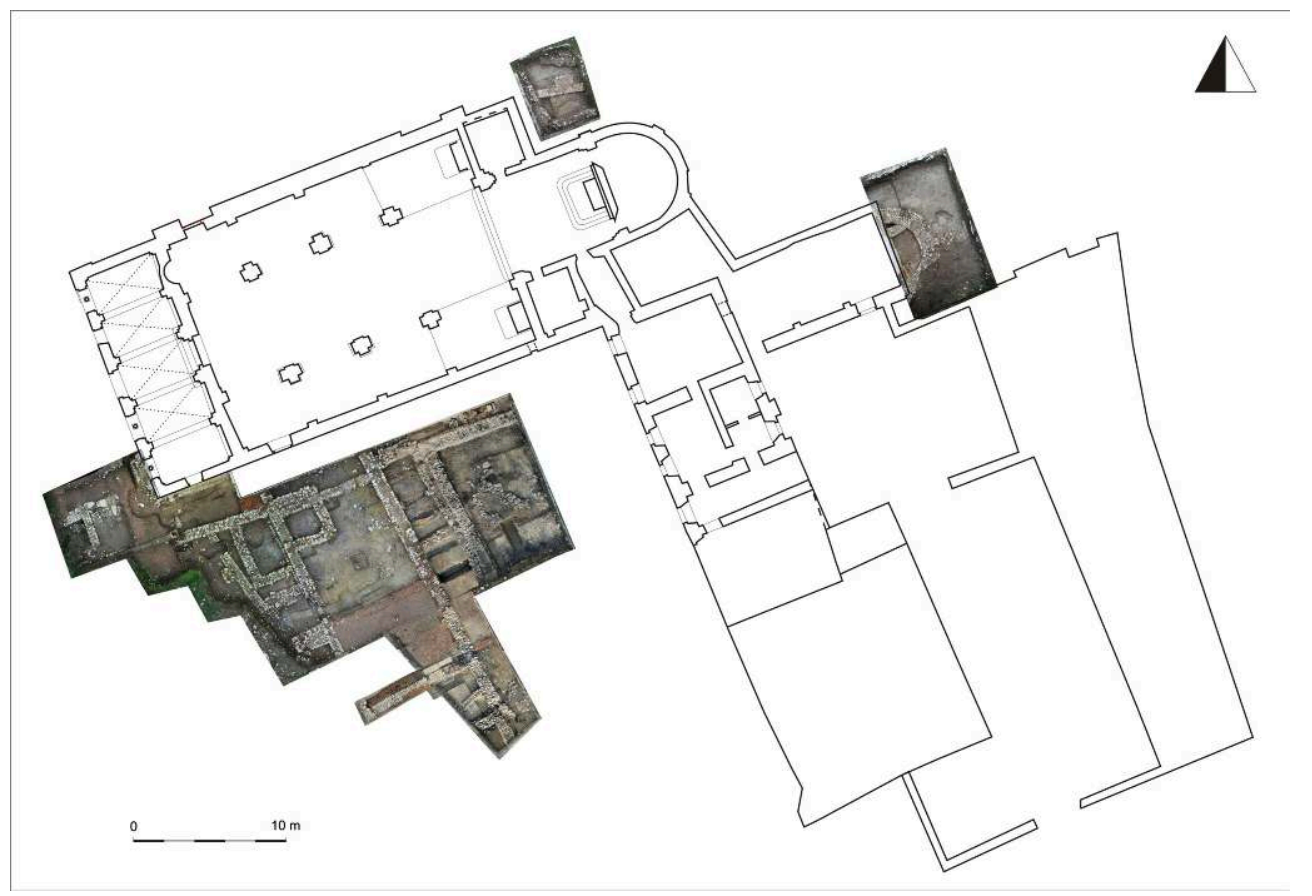


Fig. 14 - Castelletto Cervo. Portail sur le côté ouest de l'aile orientale du cloître, possible accès à la salle capitulaire (cl. E. Destefanis).

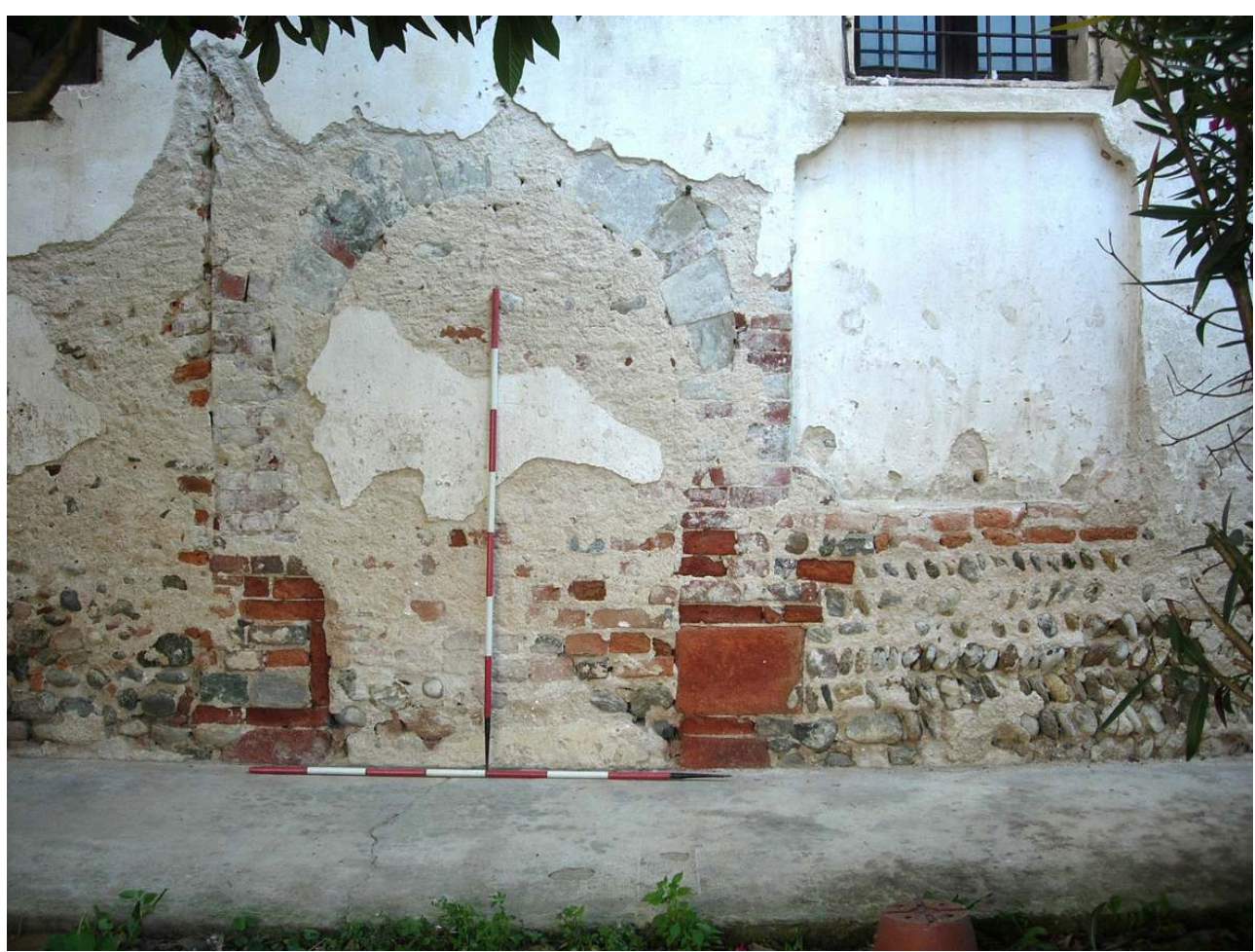

Dans l'aile orientale, le côté ouest montre, sous plusieurs couches d'enduit qui ne laissent entrevoir que très partiellement la maçonnerie, un portail avec un arc en pierre de taille et quelques briques qui, par sa position, semblerait correspondre à l'ouverture de la salle capitulaire (fig. 14). Il était peut-être flanqué par deux ouvertures symétriques, selon le schéma bien connu pour les salles du chapitre. Un renfoncement moderne sur la droite, surmontant une bande de galets en opus spicatum, très proche des maçonneries romanes du site, pourrait rappeler la position de la fenêtre méridionale, tandis que sur la gauche, un bouchage postérieur garde un petit claveau arrondi en pierre de taille (indice d'une baie de la salle capitulaire ?), tout à fait semblable à ceux que l'on voit dans les baies de la façade de l'église et, bien que plus rares, encore dans les archivoltes des baies géminées de l'avant-nef qui va s'appuyer contre la façade même, probablement dans la seconde moitié du XII siècle ou dans les premières décennies du siècle suivant.

Contre l'aile orientale du cloître, vers l'est, s'appuie un autre édifice, qui vient en même temps condamner une baie en briques que l'on entrevoit sous l'enduit dans le mur gouttereau est du bâtiment claustral. Il se présente assez remanié, surtout à l'intérieur (il a été utilisé comme dépôt et cave au moins dès le XIX siècle), et il est identifiable avec la chapelle mentionnée dans la documentation d'époque moderne sous la dédicace de Sainte-Marie ${ }^{58}$. 
Fig. 15 - Castelletto Cervo, église Sainte-Marie. Sur la gauche : mur gouttereau sud ; sur la droite : plan de l'église (état actuel) et proposition de reconstruction du côté sud (cl. et élaboration E. Destefanis).

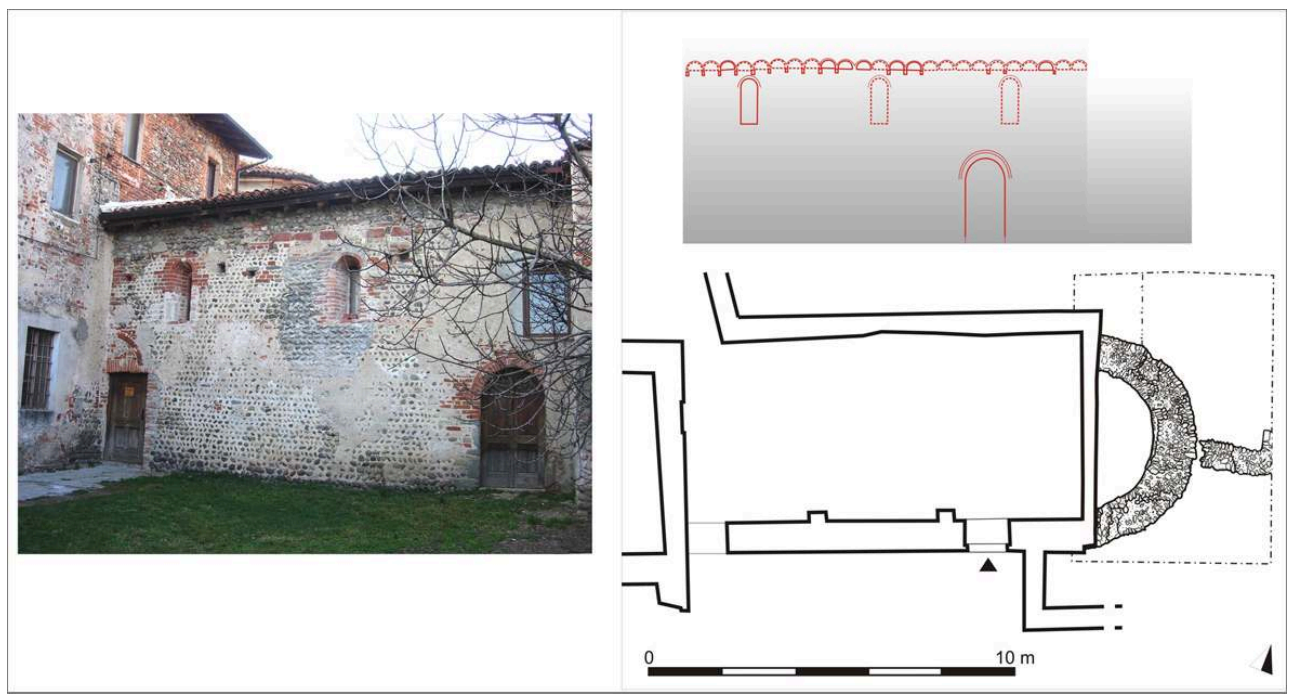

Elle garde, pour ce qui concerne la période la plus ancienne, le mur gouttereau sud (fig. 15), en galets disposés en opus spicatum et une corniche continue à arcatures en briques, remplies par des dalles en demi-cercle en brique et en pierre, qui ont partiellement survécu. La fouille menée à l'extérieur, vers l'est, au-delà du mur droit d'époque moderne qui ferme actuellement le bâtiment, a permis de mettre en évidence la fondation complète de l'abside, dont subsiste encore un petit morceau en élévation, qui pourrait suggérer un mur scandé par des lésènes, portant, peut-être, la même corniche que l'on voit sur le côté sud. Les dimensions que l'on peut ainsi restituer sont de $14,30 \mathrm{~m}$ de longueur sur $6 \mathrm{~m}$ de largeur environ. À l'intérieur, le même mur méridional conserve partiellement des demi-piliers en brique en alternance avec des blocs en pierre de taille, qui soutiennent une voûte en berceau apparemment d'origine (fig. 16). La communication entre cet édifice et le chapitre est assurée par un portail en pierre de taille qui s'ouvre dans le mur est du bâtiment oriental du cloître, désaxé vers le nord par rapport au portail de la possible salle capitulaire : des remaniements tout à fait récents ont compromis la maçonnerie ancienne, mais il semble que l'on peut déceler que le portail s'insère dans la maçonnerie précédente, ce qui confirmerait la postériorité de la deuxième église. 
Fig. 16 - Castelletto Cervo, église Sainte-Marie. Vue de l'intérieur (cl. E. Destefanis).

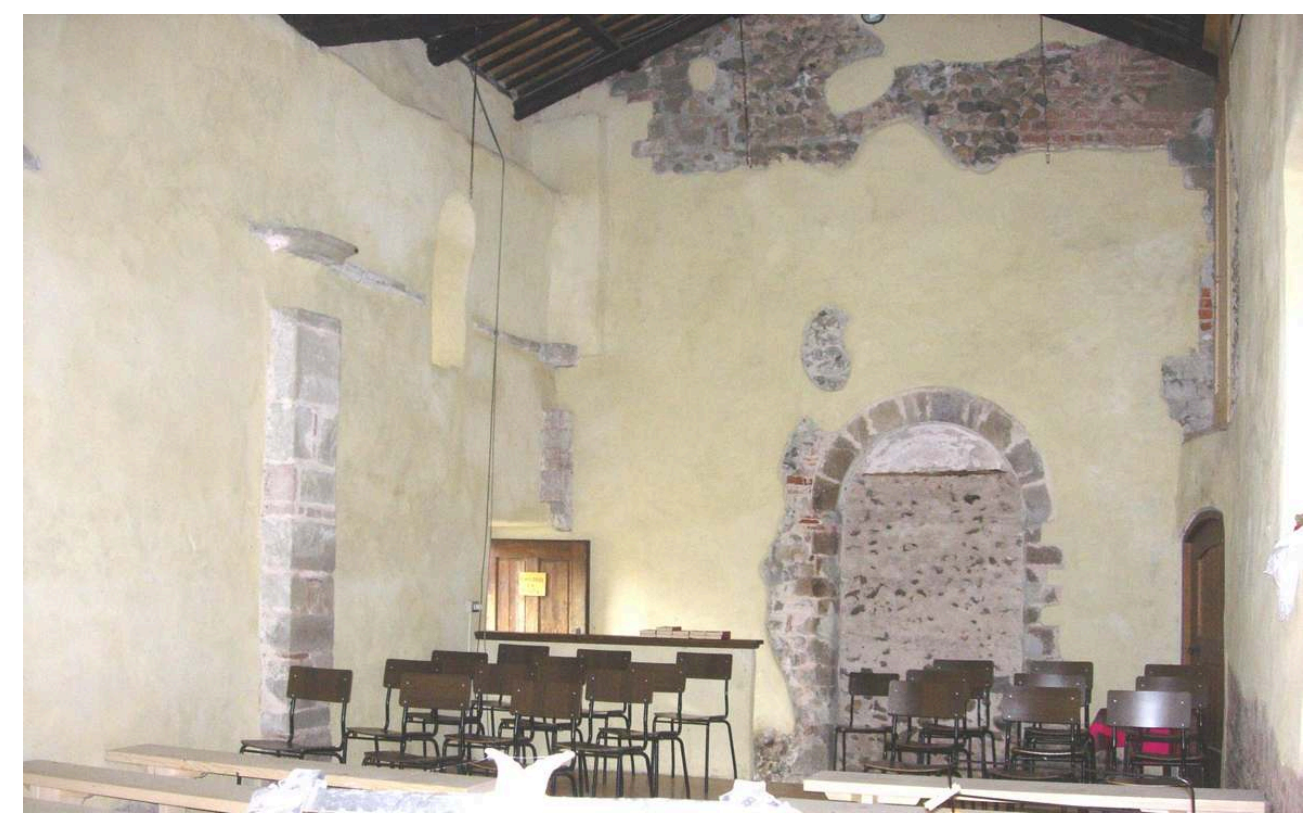

Un autre portail s'ouvre dans le mur sud; seule la baie de gauche subsiste dans un état quasi d'origine, l'autre ayant été restaurée (mais apparemment à l'emplacement de l'ancienne). La chronologie reste pour le moment à préciser, dans l'attente aussi des résultats des analyses de thermoluminescence. Les caractères stylistiques et les rapports stratigraphiques sembleraient pour l'instant conduire vers le milieu du $\mathrm{XII}^{\mathrm{e}}$ siècle ${ }^{59}$, ce qui empêcherait de reconnaître dans cette chapelle, comme on l'a vu, la première église du monastère. Les ressemblances avec la situation reproduite dans le plan d'Argon sont évidentes, mais on en retrouve aussi un écho à Vertemate, qui partage d'ailleurs un prieur avec Castelletto, vers la fin du XI ${ }^{e}$ siècle ${ }^{60}$.

39 À l'occasion de la fouille de 2012, qui s'est concentrée en partie sur le secteur oriental de l'ensemble, à côté (vers le sud) de l'église mariale, on a pu mettre en évidence les fondations d'un mur nord-sud qui vient s'appuyer au mur gouttereau méridional de la chapelle. Cette maçonnerie est conservée en élévation seulement dans sa partie nord, tandis que pour le reste elle ne subsiste qu'en fondation. La fonction de cette structure, contre laquelle vient s'appuyer un bâtiment du bas Moyen Âge, reste pour l'instant à préciser : elle semble en tout cas délimiter un espace (cour ?), ayant sur les autres côtés la chapelle mariale et l'aile est du cloître. 
Fig. 17 - Castelletto Cervo. Porte médiévale bouchée et mur qui s'appuie au sud contre l'église Sainte-Marie (cl. E. Destefanis).

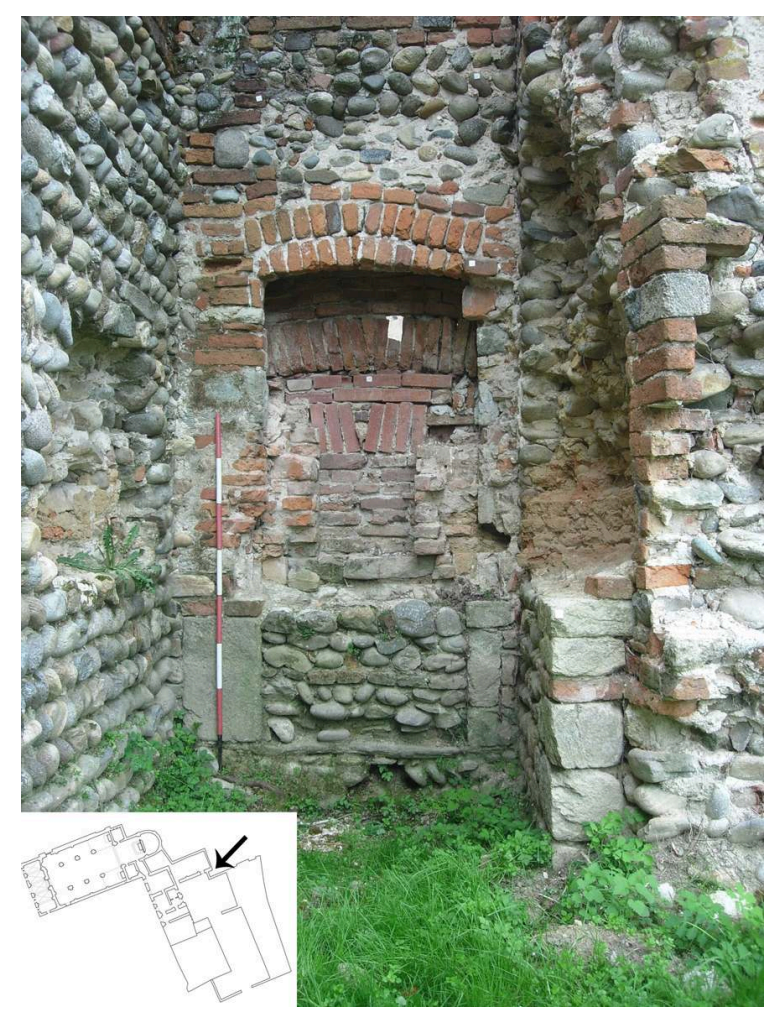

Le parement conservé tout près de l'église (fig. 17) montre plusieurs phases et il est très perturbé par des interventions modernes (un four a été installé dans ce coin). La partie haute, à cause de sa technique de construction, ne semble pas remonter avant le bas Moyen Âge, tandis qu'en dessous de cette bande tardive, une maçonnerie plus ancienne est préservée. Elle est percée par une porte, dont on observe encore très nettement le seuil en pierre et les montants (bien que celui de droite ait été l'objet, à son tour, de remaniements postérieurs), réalisés dans une technique assez proche de celle que l'on voit dans l'église Sainte-Marie, avec une alternance de pierre de taille et de brique.

41 Il est difficile, en l'état actuel, de préciser l'organisation architecturale et la fonction de cette partie du monastère, bien que la comparaison avec le plan d'Argon demeure séduisante. Les deux ouvertures de la chapelle et celle mise en évidence dans le mur nord-sud dont on vient de parler laissent d'ailleurs entrevoir une circulation complexe qui se met en place progressivement dans ce secteur du prieuré, dont la correspondance (même partielle) avec la liturgie clunisienne reste une question à laquelle il convient de réfléchir.

42 Enfin, pour compléter l'emplacement de l'église secondaire, il faudrait également considérer l'espace ouvrant sur son côté septentrional et oriental et qu'on a pu fouiller sur une zone très réduite. Néanmoins, la mise en évidence (bien que partielle) de trois sépultures - qui laissent supposer une aire funéraire plus étendue -, situées immédiatement à l'est de l'abside, appartenant apparemment à la même phase et qui semblent pouvoir être datées, du moins en partie, de la seconde moitié du XII ${ }^{\mathrm{e}}$ $\mathrm{XIII}^{\mathrm{e}}$ siècle ${ }^{61}$, pose évidemment une série de questions sur le rapport entre cette chapelle et l'aire d'inhumation environnante, aussi bien au niveau des passages et de la 
communication entre ces deux espaces que sur ses fonctions funéraires, qui apparaissent de plus en plus multiples et nuancées ${ }^{62}$.

Il est évidemment impossible de tirer des conclusions des données sur la situation nord-italienne en l'état actuel, notamment pour ce qui concerne les dérivés clunisiens, dans l'attente de connaître plus en détail la situation de Cluny. On ne se bornera donc ici qu'à quelques réflexions.

En général, la présence d'une église secondaire, toujours attestée assez tardivement par les sources et documentée au niveau des structures matérielles pour le XII ${ }^{\mathrm{e}}$ siècle seulement dans les cas de Piona et de Castelletto, n'est pas commune. Faute de fouilles et d'analyses du bâti réalisées de façon systématique, il n'est pas possible pour l'instant de vérifier, pour la plupart des prieurés, si cet édifice n'a effectivement jamais existé ou s'il n'apparaît pas dans la documentation, textuelle ou archéologique, dont on dispose pour l'instant. En admettant parfois une absence effective, la question qui s'impose est de savoir si dans ces cas, les exigences liturgiques sont moins complexes et que par conséquent, on n'a pas besoin d'avoir une chapelle si dense de significations et de fonctions ou si, en revanche, ces fonctions sont associées à d'autres espaces du monastère. C'est le problème que l'on vient d'exposer à propos de la chapelle mariale dans un des collatéraux de la prieurale, documentée dans plusieurs prieurés, selon l'interprétation proposée par Paolo Piva. En tout cas, au moins dans l'état actuel des connaissances, ces édifices sont présents seulement dans les prieurés et pas dans les dépendances, ce qui pourrait témoigner ultérieurement d'une complexité de la vie liturgique bien différente par rapport au différent statut du site. L'idée suggérée par Piva à propos de Piona, de la réalisation de la chapelle mariale en rapport avec l'acquisition du status de prieuré ou, au moins, avec une progressive affirmation de cette institution, semble aller dans cette direction.

D'ailleurs, si l'on revient sur le cas de Castelletto, dont la qualification de prieuré n'est pas discutée, on voit que le monastère va s'organiser, dans sa topographie et ses édifices, au fil des décennies: l'église secondaire ne semble pas une construction d'origine, mais s'inscrit plutôt dans un processus «d'agrégation » de bâtiments au noyau central, représenté par l'église prieurale et par le cloître. Ce processus, intéresse surtout le $\mathrm{XII}^{\mathrm{e}}$ siècle et probablement la première moitié du suivant - et coïncide avec un moment de grande prospérité au niveau économique et patrimonial ${ }^{63}$. Il répond sans doute à une volonté de monumentalisation et s'intègre strictement aux exigences d'une vie liturgique assez riche et complexe (rehaussement de la nef centrale, construction de la partie haute du clocher, avec des fenêtres trilobées à colonnettes aux chapiteaux et tailloirs sculptés) ${ }^{64}$. La réalisation de l'avant-nef devant la façade de l'église, dont les nombreuses fonctions (à signification symbolique) sont aujourd'hui mieux connues en milieu clunisien ${ }^{65}$, et, peut-être un peu avant, de la chapelle mariale, semble en témoigner clairement.

46 Le cas de Castelletto, mais aussi pour certains aspects celui d'Argon (bien que d'une façon différente, liée à la nature de la documentation), montre la nécessité de réinsérer cette chapelle dans son contexte. Le rapport avec les bâtiments environnants est encore très peu connu pour les prieurés italiens et l'examen de cet élément ne peut se faire qu'en considérant ses relations avec le monastère dans sa totalité. Les cas dernièrement cités, auxquels il faudrait ajouter celui de Vertemate, confirment la disposition "clunisienne", prévoyant un contact, dans ces cas directs, entre la deuxième église et la salle capitulaire, par le moyen d'un portail ouvert dans le mur est 
de celle-ci, légèrement désaxé par rapport à l'entrée vers le cloître, probablement afin de préserver l'emplacement du siège du prieur au centre.

En même temps, vers le côté oriental de l'ensemble, la chapelle est en relation avec d'autres espaces bâtis, occupés à Cluny par l'infirmerie, un rapport qui explique aussi une partie des fonctions que cette ecclesia remplit en milieu clunisien, au service des membres malades ou qui ne peuvent pas être intégrées complètement dans la communauté ${ }^{6}$. Pour l'instant, le cas de Castelletto, avec le portail sur le gouttereau sud de l'église et la porte dans le mur nord-sud tout à côté, peut fournir quelques traces d'une organisation complexe, à la fois des espaces et des parcours, autour de la petite cour orientale, organisation que le plan d'Argon permet de mieux visualiser.

C'est donc seulement en gardant à l'esprit ce contexte - et en poursuivant la recherche (surtout archéologique, dans les sites où les conditions sont encore réunies pour le faire) dans la direction précise de l'étude des ensembles - que l'on pourra peut-être mieux évaluer la question concernant le degré d'adhésion des prieurés italiens à Cluny, en considérant très ponctuellement, au-delà des aspects morphologiques des édifices de culte, la chronologie, le phasage, l'organisation de l'espace, la circulation, du point de vue de la liturgie mais aussi de la vie quotidienne des moines.

\section{NOTES}

1. Parmi les contributions fondamentales sur ce thème, cf. Cluny in Lombardia, Actes du Colloque (Pontida, 22-25 avril 1977), Cesena, 1979 et 1981 (Appendici e Indici) (Italia Benedettina, 1); C. VIOLANTE, A. SPICCIANI, G. SPINELl (dir.), L'Italia nel quadro dell'espansione europea del monachesimo cluniacense, Actes du Colloque international (Pescia, 26-28 novembre 1981), Cesena 1985 (Italia Benedettina, 8) ; E. CAU, A. A. SETTIA (dir.), San Maiolo e le influenze cluniacensi nell'Italia del Nord, Actes du Colloque international (Pavia-Novara, 23-24 septembre 1994), Como, 1998 (Biblioteca della Società Pavese di Storia Patria, n.s., 7); G. ANDENNA «I priorati cluniacensi in Italia in età comunale (secoli XI-XIII)», G. CONSTABLE, G. MELVILLE, J. OBERSTE (dir.), Die Cluniazenser in ihrem politisch-sozialen Umfeld, Münster, 1998 (Vita Regularis, 7), p.495-502; ID., Sanctimoniales cluniacenses. Studi sui monasteri femminili di Cluny e sulla loro legislazione in Lombardia (XI-XV secolo), Münster, 2004 (Vita Regularis, 20). Pour une récente synthèse je me permets de renvoyer à : E. DESTEFANIS, Le monachisme clunisien dans l'Italie du Nord ( $x^{e}$ siècle-première moitié XII ${ }^{e}$ ), in Cluny, le monachisme et la société au premier âge féodal (880-1050), Actes du Colloque international (Romainmôtier, 24-26 juin 2010), sous presse. Je renvoie à cette bibliographie générale pour le contexte auquel je fais référence dans le texte qui suit.

2. P. PIVA, Architettura monastica nell'Italia del Nord. Le chiese cluniacensi, Milano, 1998; ID., «L'architettura dei cluniacensi da Cluny all'Europa », in R. CASSANELLI, E. LóPEZ-TELLO GARCíA (dir.), Benedetto, L'eredità artistica, Milano, 2007 ; A. SEGAGNI MALACART, "Cluny en Lombardie", in N. STRATFORD (dir.), Cluny onze siècles de rayonnement, Paris, 2010, p. 328-346.

3. Les fouilles récentes semblent toutefois dès maintenant montrer une situation plus articulée et complexe par rapport aux reconstructions de l'archéologue américain, très conditionnées par le Liber Tramitis et les coutumiers clunisiens. Cf., sur ce problème : A. BAUD, G. ROLLIER, « Liturgie et espace monastique à Cluny à la lecture du Liber Tramitis, 'Descriptione monasterii' et données 
archéologiques ", in A. BAUD (dir.), Espace ecclésial et liturgie au Moyen Âge, Actes du Colloque international (Nantua, novembre 2006), Lyon, 2010 (Travaux de la Maison de l'Orient et de la Méditerranée, 23), p. 27-42 et, pour les nouvelles données archéologiques: ch. SAPIN, A. BAUD, "Cluny sous les feux de l'archéologie du bâti : les campagnes de fouilles et d'études, 2006-2011 ", in Bulletin Monumental, 2012, 2, p. 18-21; cf. aussi les contributions de A. BAUD et ch. SAPIN dans ce volume.

4. Il suffit de considérer, encore tout récemment, la perspective de M. BERETTA, «L'abbazia di San Giovanni Battista a Vertemate e l'espansione dei modelli cluniacensi », in A. C. QUINTAVALLE (dir.), Medioevo: $i$ modelli, Actes du Colloque International (Parma, 27 septembre-1 octobre 1999), Milan, 2002, p. 516 : en reconnaissant à Vertemate une première phase de l'église caractérisée par un plan en " $T$ ", tel que les reconstructions de Conant montrent pour la deuxième phase de l'église Sainte-Marie, Beretta souligne comment « l'utilizzo della pianta a $\mathrm{T}$ a Vertemate non sia casuale, ma sia da mettere in relazione proprio allo stretto legame che intercorreva tra il priorato e Cluny » (p. 516).

5. PIVA, Architettura, op. cit., p. 11-13.

6. SEGAGni MALACART, Cluny, loc. cit., part. p. 331.

7. PIVA, Architettura, op. cit., p. 14.

8. Sur ce personnage et ses rapports avec le monde clunisien, cf. C. VIOLANTE, "Per una riconsiderazione della presenza cluniacense in Lombardia ", in Cluny in Lombardia (1981), op. cit., p. $577-580$.

9. G. SPINELLI, « Gerardo della Charité e Gerardo di Vertemate: un'unica personalità storica? », in CONSTABLE, MELVILLE, OBERSTE (dir.), op. cit., p. 523-536. Piva (cf. note suivante) rappelle aussi le rôle de David, prieur d'Argon.

10. PIVA, L'architettura dei cluniacensi, loc. cit., p. 184 ("Ciò non significa che svolgessero il ruolo di 'architetti', ma di committenti che esigevano la traduzione materiale di 'spazi funzionali' giudicati irrinunciabili per quanto suscettibili di 'varianti' »).

11. PIVA, Architettura, op. cit., p. 145. En particulier, cet auteur parle d'une « utilizzo simultaneo di una tipologia 'a uso priorati' (coordinamento nuovo di vecchie formule monastiche) ».

12. Cette disposition est considérée par Neil Stratford comme typiquement clunisien (avec l'avant-nef qui précède la façade de l'église principale et sur lequel donne directement le cloître). Cf. N. STRATFORD, « Existe-t-il un art clunisien?", in Cluny. Onze siècles, op. cit., p. X.

13. N.REVEYron, «Marcigny, Paray-le-Monial et la question de la chapelle mariale dans l'organisation spatiale des prieurés clunisiens $\mathrm{au} \mathrm{XI} \mathrm{I}^{\mathrm{e}}-\mathrm{XII}{ }^{\mathrm{e}}$ siècle ", in Viator, 41 (2010), p. 63-94 (p. 63 pour la citation) : «Il faut entendre par «édifice identitaire » une architecture qui, dans son plan et sa localisation reflète l'identité d'une institution religieuse, par le biais de la spiritualité ... et des pratiques liturgiques qui lui sont attachées ». Avec cette contribution, nous renvoyons également à des réflexions plus générales sur les aspects topographiques, liturgiques et fonctionnels des chapelles mariales dans le monde clunisien. Cf. ID., «Hugues de Semur et le projet du grand Cluny», in A.C. QuinTAVAlle (dir.), Medioevo: $i$ committenti, Actes du Colloque international (Parma, 21-26 septembre 2010), Milano, 2011, p. 258-267.

14. Cf. C. MARCoRA, Il Priorato di Piona, Lecco, 1972, p. 31-33 ; ID., «I cluniacensi in diocesi di Como », in Cluny in Lombardia (1979), op. cit., p. 259-260 et PIVA, Architettura, op.cit., p. 93, les deux avec références documentaires. À ce dernier ouvrage (p. 93-96) se rapportent les observations sur le site ci-dessous, sauf indication différente.

15. Une inscription encore partiellement visible sur le mur nord de l'église au début du $\mathrm{XX}^{\mathrm{e}}$ siècle, mais dont la transcription reste très douteuse (ainsi que sa datation à de 1138), relate une dédicace à la Vierge, qui aurait pu, selon Piva, être suivie par l'énumération d'autres saints, parmi lesquels le dédicataire saint Nicolas, dans la partie non conservée du texte épigraphique. 
16. Archives d'État de Como, Fondo U.T.E., Catasto teresiano, Mappe, Olgiasca con Piona, cart. 117, mappa di prima stazione (Piona), $\mathrm{f}^{\circ}$ 3-4 ; mappa di seconda stazione, $\mathrm{f}^{\circ} 1$.

17. En 1593, la visite pastorale de l'évêque F. Feliciano Ninguarda, relate que « dentro da una parte del monastero, doppo li muri della capella magiore, si va in una capella di S. Maria antichissima ». Cf. A. GIUSSANI, «Il priorato di S. Nicolò di Piona dopo i restauri », in Rivista archeologica della provincia e antica diocesi di Como, 56-57-58 (1908), p. 64.

18. MARCoRA, Il Priorato, op. cit., p. 61. Cavagna Sangiuliani, en revanche, parle d'une dizaine de mètres (ibid., p. 30, n. 23).

19. PIVA, Architettura, op. cit., p. 96.

20. PIVA, Architettura, op. cit., p. 95-96 met en évidence la rareté de cette situation - sûrement due aux contraintes du terrain rocheux - en milieu clunisien, qui montre en revanche l'attention particulière portée à la communication aisée et directe entre la deuxième église et la salle du chapitre-chœur de l'édifice principal.

21. Sur le cadastre, le mur gouttereau est de cette aile du cloître est représenté par une simple ligne rouge, ce qui pourrait indiquer que, à cette époque, cette partie du bâtiment était déjà en ruine.

22. On se réfère pour ce site à l'analyse très détaillée dans P. PIVA, «Architettura dei cluniacensi in alta Italia: San Paolo d'Argon ", in F. G. B. TROLESE (dir.), Il monachesimo italiano nell'età comunale, Actes du IV Colloque d'Études Historiques sur l'Italie bénédictine (Pontida, 3-6 septembre 1995), Cesena, 1998, p. 317-345.

23. Recueil des chartes de l'Abbaye de Cluny, A. BERNARD, A. BRUEL (éd.), IV, Paris 1888, doc. n 3536 , p. 657-660.

24. Cf. infra pour la discussion de ce point.

25. Recueil, op. cit., IV, doc. $n^{\circ}$ 3606, p. 765-768.

26. Sur le site et pour les considérations qui suivent: PIVA, Architettura, op. cit., p. 68-73. Une interprétation différente, concernant notamment le phasage de l'église prieurale - où l'on distingue une première phase avec une seule nef débouchant sur le transept et le sanctuaire actuels, et une deuxième qui correspond à l'édifice à trois nef actuel - a été récemment fournie par BERETTA, L'abbazia, loc. cit., qui reprend essentiellement la reconstruction de l'arch. Clemente Bernasconi, responsable des travaux de restauration effectués sur le site pendant les années soixante $\mathrm{du} \mathrm{xx}^{\mathrm{e}} \mathrm{s}$. Cette reconstruction montre des problèmes qu'on ne peut pas toutefois discuter ici.

27. Recueil, op. cit., IV, $\mathrm{n}^{\circ} 3863$, p. 213-215. Sur le prieuré et son architecture cf. PIVA, Architettura, op. cit., p. 98-101.

28. Liber Notitiae Sanctorum Mediolani, M. MAGISTRETTI, U. MONNERET DE VILLARD (éd.), Milano, 1917, col. 227A (« in monasterio de Figina altare santi Martini »), 261D («altare sancte Marie in monasterio de Figina »).

29. Ibid. col. 356A (« loco Figina monasterio, ecclesia sancti Sigismundi »).

30. R. BERETTA, « Il Priorato cluniacense di S. Nicolao in Figina di Villa Vergano », in Archivio Storico Lombardo, 62 (1935), p. 99.

31. L'emplacement précis de cette église reste toutefois assez incertain: le même Beretta rappelle d'ailleurs l'existence d'un cloître, situé «a destra [de l'église, n. de l'A.] con cortile quadrato a colonne » (BERETTA, Il Priorato, loc. cit., p. 100). Bien que remanié par rapport à l'époque romane, mais encore de quelque façon visible dans la première moitié du $\mathrm{xx}^{\mathrm{e}}$ siècle, ce cloître placé au sud de l'église emmènerait à exclure le développement du cimetière sur ce côté et à penser, en revanche, à l'emplacement de la chapelle de Saint-Sigismond au nord du bâtiment de culte principal. 
32. A. BONAVITA, M. GALli, M. LEONI, Vertemate con Minoprio. L'abbazia, il castello, la villa, Como, 2009, p. 20 (visites des évêques Ninguarda et Archinto, 1592 et 1597, qui relatent la présence de cet autel dans le collatéral nord).

33. PIVA, Architettura, op. cit., p. 100, où l'auteur rappelle aussi les cas de Vallate et d'olgiate Comasco.

34. Recueil, op.cit., IV, $\mathrm{n}^{\circ} 3603$, p. 762-764.

35. A. BREDA, A. VALSECCHI, «San Pietro in Lamosa. La vicenda architettonica tra Medioevo e Rinascimento ", in F. SINA, A. VALSECCHI, San Pietro in Lamosa in Proviaglio d'Iseo. Storia e arte, Provaglio d'Iseo, 2007 ( $2^{\mathrm{e}}$ éd., $1^{\mathrm{e}}$ éd. Provaglio d'Iseo, 2004), p. 15-39. Pour les fouilles dans la galerie nord du cloître actuel: I. VENTURINI, "Provaglio d'Iseo (BS) 2008. Monastero di S. Pietro in Lamosa. Accertamenti archeologici nel claustrum », rapport de fouille près de la Soprintendenza per i Beni Archeologici della Lombardia-Ufficio di Brescia. Je remercie M. Dr. Andrea Breda de la même Soprintendenza, directeur scientifique de la fouille, qui a permis la consultation de ce rapport.

36. PIVA, Architettura, op. cit., p. 67.

37. M. G. MORI, « La decorazione pittorica dei secoli XIV e XVI ", in SINA, VALSECCHI, op.cit., p. 63 (dans le même texte on propose toutefois aussi une datation au XI ${ }^{\mathrm{e}}$ siècle).

38. É. PALAZzo, « Marie et l'élaboration d'un espace ecclésial au haut Moyen Âge », in D. IOGNA-PRAT, É. PALAzzo, D. Russo (dir.), Marie. Le culte de la Vierge dans la société médiévale, Paris, 1996, p. 313-325, en particulier p. 315-316 (d'où sont tirées les citations).

39. Archives de la Paroisse de Castelletto Cervo, Inventari, perizie, stato d'anime, note beni Parrocchia SS. Pietro e Paolo dal 1668 al 1945, mons. Costa d'Arignano, rapport pour la visite pastorale, 5 août 1770. Le rapport relate que dans l'église, désormais paroissiale, il y a trois autels : le principal, dédiés aux Apôtres, un autre de la Vierge et un troisième (sûrement d'époque moderne) dont les dédicataires sont saint Charles et saint Gratus. Une statue en bois de la Vierge, probablement à dater à l'époque médiévale (mais assez remaniée à la période moderne) et appartenant peut-être dès l'origine à l'église principale, est conservée encore aujourd'hui par le Curé.

40. Recueil, op. cit., IV, $\mathrm{n}^{\circ}$ 3548, p. 675-677.

41. PIVA, Architettura, op. cit., p. 56-63 ; M. SPINI (dir.), S. Egidio di Fontanella al Monte. Le vicende storiche e costruttive del priorato Cluniacense (secoli XI-XII), vol. 2, Comune di Sotto il Monte Giovanni XXIII, 2001 ; SEGAGNI, Cluny, loc. cit., p. 333.

42. M. tagliabue, L. Chiodi, Il priorato di S. Egidio dei Benedettini Cluniacensi in Fontanella del Monte (1080-1473). Storia e documenti, Bergamo, 1960, doc. n 1, p. 79.

43. PIVA, Architettura, op. cit., p. 56-57.

44. TAGLIABUE, CHIODI, Il priorato, op. cit., doc. $\mathrm{n}^{\circ} 1$, p. 79.

45. TAGLIABUE, CHIODI, Il priorato, op. cit., doc. $n^{\circ} 8 / 1$, p. 108-109: «Alla qual gesia a mezzodì è un claustro piccolo, et contiguo alla gesia, qual claustro va in ruina; una capelletta et in essa il sepolcro di S. Toperga dove si dice esser anco il suo corpo [...] Et alli detti capella, et sepolcro è aderente un loco qual si chiama capitolo, et un torchio subsequente a detta corte [...]».

46. SPINI, S. Egidio, op. cit., 2, doc. $\mathrm{n}^{\circ} 14$, p. 26: «Item el Claustro contiguo a la gesia dentro lo Claustro una Capella de S. Toperga dove se dise esser il corpo de la ditta Sancta ».

47. SPINI, S. Egidio, op. cit., 1, p. 19.

48. C'est la position que le sépulcre occupe dès le début $d u \mathrm{xvII}^{\mathrm{e}}$ siècle, quand une visite de l'agent des Procuratori di S. Marco, en 1604, relate : «Apresso la porta picciola di chiesa che va nell'inclaustro a man manca vi è il sepolcro di Santa Teuperga [...] facendosi, dopo andato quel Priorato in commenda, della cappelletta, et del capitolo, maghazeni [...] fu traslato il corpo santo fuori della cappelletta ». Le monument a été placé dans sa position actuelle, contre le mur sud de l'église, mais bien plus à l'ouest, seulement depuis la seconde moitié du xx siècle (sPINI, S. Egidio, op. cit., 1, p. 20). 
49. Même pour Pontida Piva (Architettura, op.cit. p. 41) a supposé la présence d'une chapelle ayant pu fonctionner comme un deuxième chœur monastique « collegato al capitolo o comunque prossimo al santuario della chiesa maggiore", comme l'église du cimetière ou bien encore comme une chapelle funéraire pour le fondateur du prieuré, Albert da Prezzate. L'hypothèse se fonde sur une mention de 1204 d'une ecclesia S. Michaelis, qui, selon Piva, aurait pu abriter le sépulcre d'Albert, dont on conserve deux dalles sculptées où se trouve la représentation de l'archange.

50. Sur ces aspects: P. Golinelli (dir.), Storia di San Benedetto Polirone. Le origini (961-1125), Bologna, 1998.

51. Pour une synthèse, cf. P. PIVA, «L'abbazia di Polirone nel XII secolo: architettura e vita monastica. Una lettura comparata della documentazione archeologica e scritta", in A.C. QUINTAVAlLE (dir.), Arredi liturgici e architettura, Milano, 2007, p. 53-85. Concernant les rapports entre l'architecture et la liturgie polironienne, exprimée par les Consuetudines, cf. ID., Da Cluny a Polirone. Un recupero essenziale del romanico europeo, San Benedetto Po, 1980, avec une transcritption (partielle) des coutumiers.

52. A. C. QUINTAVALLE, Wiligelmo e Matilde. L'officina romanica, Milano, 1991, p. 84-125 (et part. p. 121).

53. Cf. ci-dessus.

54. Pour une synthèse sur les travaux archéologiques et une première proposition de phasage, cf. s. LUSUARDI SIENA, C. GIOSTRA, « San Benedetto Po: l'abbazia di Matilde di Canossa. Archeologia di un grande monastero dell'Europa benedettina ", in M.c. SоммA (dir.), Cantieri e maestranze nell'Italia medievale, Actes du Colloque (Chieti-San Salvo, 16-18 mai 2008), Spoleto, 2010, p. 483-498.

55. ANDENNA, Sanctimoniales, op. cit., doc. $n^{\circ} 1$, p. 156-157.

56. Pour les contributions plus récentes sur le site, cf. PIVA, Architettura, op. cit., p. 74-79 ; SEGAGNI, Cluny, loc. cit., p. 334. Pour les travaux archéologiques (fouille et archéologie du bâti), menés pendant ces dernières années par le Dipartimento di Studi Umanistici, Université du Piémont Oriental "Amedeo Avogadro", sous la direction scientifique de l'auteur de cet article: www.fastionline.org (campagnes de fouille 2009, 2010, 2011, 2012); E. D ESTEFANIS, avec la collaboration de G. ARDIZIO, E. BASSO, «Contributo alla storia del monachesimo cluniacense nell'Italia settentrionale: indagini archeologiche al priorato di Castelletto Cervo (BI) », in G.VoLPE, P. FAVIA (dir.), Atti del V Congresso Nazionale di Archeologia Medievale (Foggia-Manfredonia, 29 septembre-3 octobre 2009), Firenze, 2009, p. 492-497; G. ARDIZIO, E. DESTEFANIS, P. GREPPI, «Castelletto Cervo (Biella), ex priorato cluniacense dei SS. Pietro e Paolo. Scavo archeologico nell'area esterna alla chiesa parrocchiale ", in Quaderni della Soprintendenza Archeologica del Piemonte, 25 (2010), p. 186-189; E. DESTEFANIS, «Castelletto (Italie). Étude du bâti et analyses », in Dossiers d'archéologie, h.s. nº 19 (août 2010), Cluny et l'Europe, p. 44-47 ; E. DESTEFANIS, G. ARDIZIO, « BI, Castelletto Cervo, fraz. Garella, complesso parrocchiale dei SS. Pietro e Paolo 2009-2011", in Archeologia Medievale, 38 (2011), Notiziario, p. 333-335. Pour le contexte historique et les sources documentaires : G. ARDIZIO, E. DESTEFANIS, « Il priorato cluniacense di Castelletto Cervo (Biella) nella documentazione scritta: quadro storico e strutture materiali », in Bollettino storico-bibliografico subalpino, 109/1 (2011), p. 39-81, avec bibliographie précédente. Le site est intégré dans le Projet «Terres cuites architecturales et nouvelles méthodes de datation »(dir. P. Guibert, Ch. Sapin) et, dans ce cadre, il fait l'objet d'analyses de thermoluminescence, actuellement en cours, impliquant plusieurs édifices de l'ensemble.

57. La largeur (dimensions extérieures) des ailes orientale et occidentale est de 8,70 m.

58. Une « chiesa della Madonna » est attestée dans une visite pastorale datant de 1600 (Archives d'État de Verceil, Visite pastorali (microfilm), bob. 1, parte I, mons. Ferrero, Atti, Parrocchia di Castelletto, 15 décembre 1600); une autre visite, de 1668 (Archives de la Paroisse de Castelletto Cervo, cart. Lite Ristis, Raccolta di carteggi, Decreti di visita (...) in Castelletto l'anno 1668, 15 mai 1668) 
mentionne, après la sacristie, la " capella della Beata Vergine ivi attigua ", à laquelle est encore associée une fonction commémorative, étant le lieu où se célèbrent des messes pour les défunts qui y laissent un legs. Supprimée par disposition épiscopale en 1675 (Archives de l'Archevêché de Verceil, Visite pastorali, mons. Broglia, Atti, Parrocchia di Castelletto), la chapelle est encore nommée dans une visite pastorale en 1770, quand elle est évoquée comme "altra fabrica detta la chiesa vecchia " (Archives de la Paroisse de Castelletto Cervo, Inventari, perizie, stato d'anime, note beni Parrocchia SS. Pietro e Paolo dal 1668 al 1945, mons. Costa d'Arignano, rapport pour la visite pastorale, 5 août 1770).

59. Certains détails, tels que la corniche avec des petites demi-lunes, alternativement en briques et en pierre, entre les arcatures en briques, et, plus généralement, le goût pour la polychromie dans le choix des matériaux, tel que l'on voit aussi dans les demi-piliers à l'intérieur, sont bien connus dans la région au XII ${ }^{\mathrm{e}}$ siècle, ainsi qu'au siècle suivant. Dans l'impossibilité de développer ici le discours sur les comparaisons, je me borne à renvoyer à C. TOsco, "Architetture dei Templari in Piemonte ", in Le vie del medioevo. Pellegrini, mercanti, monaci e guerrieri da Canterbury a Gerusalemme, Actes des Colloques (Turin, 20 octobre 1994, 16 octobre 1996), Torino, 1998, p. 251 et E. DESTEFANIS, «Intorno a Fontaneto: paesaggio, insediamenti, strutture materiali nel piviere di Suno in età medievale », in G. ANDENNA, I. TERUGGI (dir.), Fontaneto: una storia millenaria. Monastero. Concilio metropolitico. Residenza viscontea, Actes des Colloques (Fontaneto d'Agogna, septembre 2007, juin 2008), Novara, 2009 (Studi Storici, 50), en particulier p. 70 et 78, avec bibliographie.

60. Le carte dell'Archivio Capitolare di Santa Maria di Novara, F. GABOTTO, G. BASSO, A. LEONE, G. B. MORANDI, o. SCARZello (éd.), II, Pinerolo, 1915 (Biblioteca della Società Storica Subalpina, 79), doc. $\mathrm{n}^{\circ}$ 277, p. $159-160$.

61. Le date provient des analyses au radiocarbone effectuées sur une des ces sépultures.

62. Cf. REVEYRON, Marcigny, loc. cit., p. 68-69, 82.

63. ARDIZIO, DESTEFANIS, Il priorato, loc.cit., p. 44-58.

64. Pour ces aspects, intégrés dans les questions plus générales concernant la vie liturgique en milieu clunisien, cf. S. Boynton, I. Cochelin (dir.), From Dead of Night to End of Day: the Medieval Customs of Cluny, Turnhout, 2005 (Disciplina monastica, 3). Cf. aussi les observations de A. RAUWEL dans ce volume. À propos des questions chronologiques, cf. les remarques de REVEYRON, Marcigny, loc. cit., p. 80-82, qui, en soulignant la connexion étroite entre la chapelle "mariale" et le cloître, dans une organisation intégrée des lieux, met en évidence le décalage, souvent attesté, de l'implantation d'édifices comme l'avant-nef ou l'église secondaire par rapport à l'ensemble église prieurale-cloître, souvent les premiers à être mis en place.

65. Sur le problème, même dans un contexte plus général des espaces occidentaux: ch. SAPIN (dir.), Avant-nefs et espaces d'accueil dans l'église entre le Ive et le XII siècle, Paris, 2002 et part. les contributions de Christian Sapin et Kristina Krüger aux p. 398-423; K. KRÜGER, Die romanischen Westbauten in Burgund und Cluny. Untersuchung zur Function einer Bauforme, Berlin, 2003 ; EAD., "Monastic Customs and Liturgy in the Light of the Architectural Evidence: a Case Study on Processions (Eleventh-Twelfth Centuries)", in BOynTon, COCHELIN, From Dead, op. cit., p. 191-219, avec bibliographie.

66. R. CRISTIANI, «Integration and Marginalization: Dealing with the Sick in Eleventh-Century Cluny », in BOYNTON, CoCHELIN, From Dead, op. cit., p. 287-295. 
INDEX

Index géographique : Italie/Castelletto, Italie/Argon, Italie/Figina, Italie/Piona, Italie/Polirone, Italie/Pontida, Italie/Provaglio, Italie/Vertemate, Italie du Nord

Mots-clés : chapelle mariale, chapitre, cloître, prieuré clunisien, circulation, architecture

\section{AUTEUR}

\section{ELEONORA DESTEFANIS}

Università del Piemonte Orientale "Amedeo Avogadro" 